Supporting Information for

\title{
Hangman Salen Platforms Containing Two Xanthene Scaffolds
}

\author{
Jenny Y. Yang, Julien Bachmann and Daniel G. Nocera* \\ Department of Chemistry, 6-335, Massachusetts Institute of Technology, 77 Massachusetts \\ Avenue, Cambridge, Massachusetts 02139-4301
}

Index Page

General Experimental Methods S2

Synthetic Scheme S1

$\mathrm{X}$-ray Structural Data for $\mathbf{9}$

S4-S14

X-ray Structural Data for 24

$\mathrm{S} 15-\mathrm{S} 22$

DFT Calculations

S23-S36

${ }^{1} \mathrm{H}$ NMR Spectra for 3, 5-14, 16, 22, 24-26

S37 
Materials. Silica gel 60 (70 - 230 and 230 - 400 mesh) was used for column chromatography. Analytical thin layer chromatography was performed using F254 silica gel (pre-coated sheets, $0.2 \mathrm{~mm}$ thick). Solvents for synthesis were reagent grade or better and used as received or dried according to standard methods. ${ }^{1}$ The following compounds were obtained using published protocols and their purity confirmed by ${ }^{1} \mathrm{H}$ NMR: 4-hydroxycarbonyl-5-bromo-2,7-ditert-butyl-9,9-dimethylxanthene (1) ${ }^{2} \quad$ 4-methoxycarbonyl-5-bromo-2,7-di-tert-butyl-9,9dimethylxanthene (2),2 4-(5-bromo-2,7-di-tert-butyl-9,9-dimethyl-9H-xanthen-4-yl)-benzoic acid methyl ester (4), ${ }^{3}$ and 4-hydroxy-biphenyl-3-carbaldehyde (21). ${ }^{4}$

Physical measurements. ${ }^{1} \mathrm{H}$ NMR spectra were collected in $\mathrm{CDCl}_{3}$ or $\mathrm{C}_{4} \mathrm{D}_{8} \mathrm{O}$ using a $500 \mathrm{~Hz}$ spectrometer at $25{ }^{\circ} \mathrm{C}$. All chemical shifts are reported using the standard $\delta$ notation in parts-per-million relative to tetramethylsilane and spectra have been internally calibrated to the monoprotio impurity of the deuterated solvent used. High-resolution mass spectral analyses were carried out using an ESI source.

1. Armarego, W. L. F.; Perrin, D. D. Purification of Laboratory Chemicals; 4th ed.; Butterworth-Heinmann: Oxford, 1966.

2. Chang, C. J.; Chng, L. L.; Nocera, D. G. J. Am. Chem. Soc. 2003, 125, 1866-1876.

3. Liu, S.-Y.; Nocera, D. G. J. Am. Chem. Soc. 2005, 127, 5278-5279.

4. Morris, G. A.; Nguyen, S. T. Tetrahedron Lett. 2001, 42, 2093-2096. 


\section{Scheme S1}

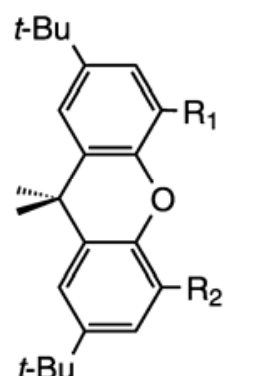

$$
\begin{aligned}
& a \square \text { 1: } \mathrm{R}_{1}=\mathrm{CO}_{2} \mathrm{H}, \mathrm{R}_{2}=\mathrm{Br} \\
& b \square \text { 2: } \mathrm{R}_{1}=\mathrm{CO}_{2} \mathrm{Me}, \mathrm{R}_{2}=\mathrm{Br} \\
& \text { 24: } \mathrm{R}_{1}=\mathrm{CO}_{2} \mathrm{Me}, \mathrm{R}_{2}=\mathrm{B}(\mathrm{OH})_{2} \\
& e \square \text { 1: } \mathrm{R}_{1}=\mathrm{CO}_{2} \mathrm{H}, \mathrm{R}_{2}=\mathrm{Br} \\
& \text { 25: } \mathrm{R}_{1}=\mathrm{CO}_{2} \mathrm{CH}_{2} \mathrm{Ph}, \mathrm{R}_{2}=\mathrm{Br}
\end{aligned}
$$

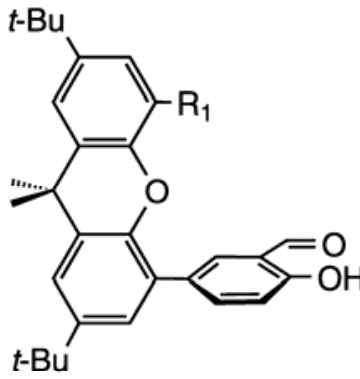

$$
\begin{aligned}
c & \multicolumn{2}{c}{24} \\
d & \text { 10: } \mathrm{R}_{1}=\mathrm{CO}_{2} \mathrm{Me} \\
& \text { 9: } \mathrm{R}_{1}=\mathrm{CO}_{2} \mathrm{H} \\
b, c & \square \text { 25 } \\
g & \text { 26: } \mathrm{R}_{1}=\mathrm{CO}_{2} \mathrm{CH}_{2} \mathrm{Ph} \\
& \text { 9: } \mathrm{R}_{1}=\mathrm{CO}_{2} \mathrm{H}
\end{aligned}
$$

(a) $\mathrm{H}_{2} \mathrm{SO}_{4}$, methanol, (b) bis(pinacolato)diboron, $\mathrm{Pd}(\mathrm{dppf}) \mathrm{Cl}_{2}, \mathrm{KOAc}$, DMSO, (c) 5-bromosalicyaldehyde, $\mathrm{Pd}\left(\mathrm{PPh}_{3}\right)_{4}, \mathrm{Na}_{2} \mathrm{CO}_{3}, \mathrm{DMF} / \mathrm{H}_{2} \mathrm{O}$, (d) $\mathrm{BBr}_{3}, \mathrm{CH}_{2} \mathrm{Cl}_{2}$, (e) benzyl alcohol, DMAP, DCC, $\mathrm{CH}_{2} \mathrm{Cl}_{2},(\mathrm{~g}) \mathrm{H}_{2}$ on $\mathrm{Pd} / \mathrm{charcoal}$ ethyl acetate 


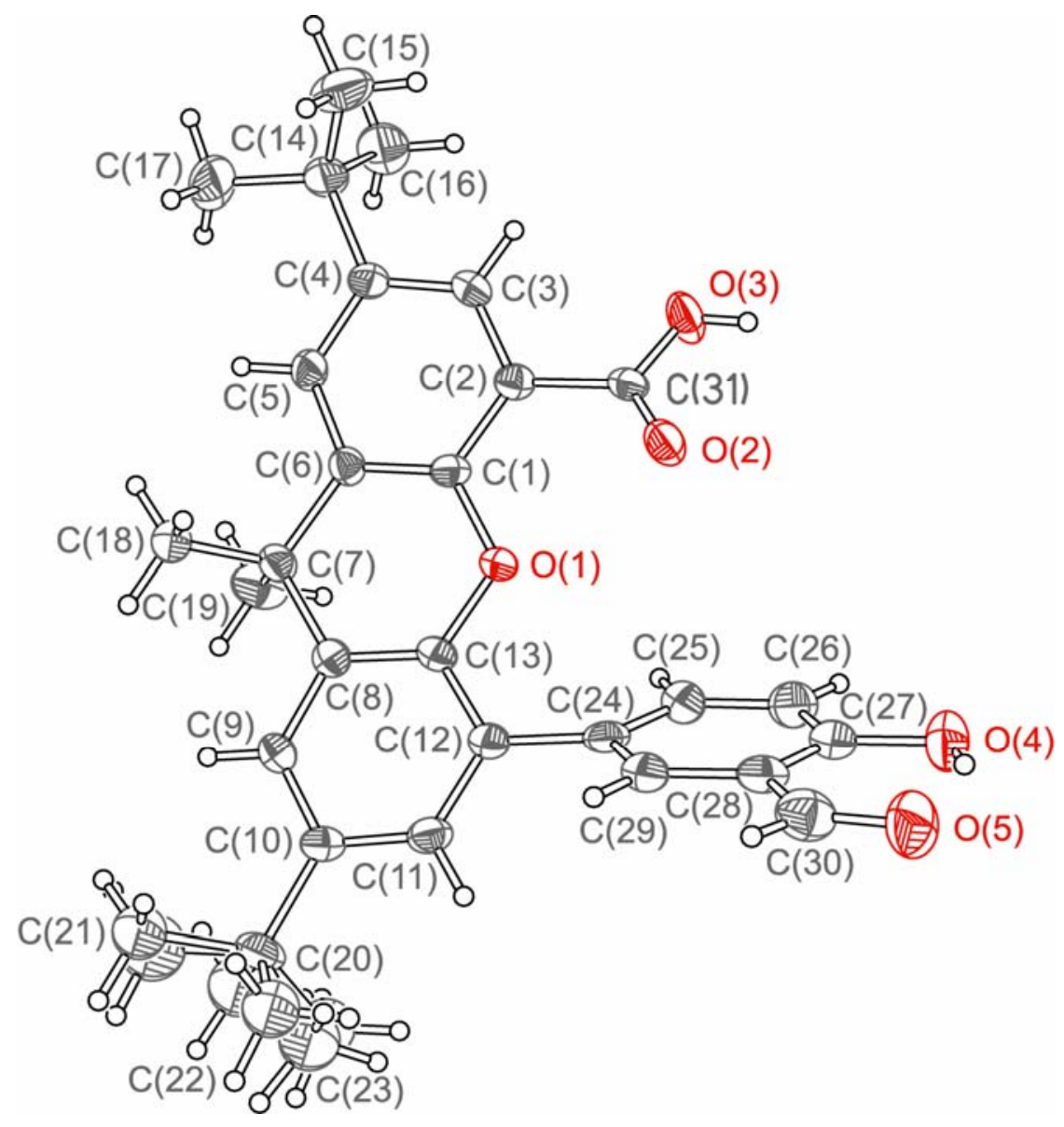

Figure S1. Thermal ellipsoid plot of 2,7-di-tert-butyl-5-(3-formyl-4-hydroxyphenyl)-9,9dimethyl-9H-xanthene-4-carboxylic acid (9). Thermal ellipsoids are drawn at the 50\% probability level. 
Table S1.1. Crystal data and structure refinement 2,7-di-tert-butyl-5-(3-formyl-4-hydroxyphenyl)-9,9-dimethyl-9H-xanthene-4-carboxylic acid (9).

\begin{tabular}{|c|c|c|}
\hline Identification code & 03084a & \\
\hline Empirical formula & C31 H34 O5 & \\
\hline Formula weight & 486.58 & \\
\hline Temperature & $467(2) \mathrm{K}$ & \\
\hline Wavelength & $0.71073 \AA$ & \\
\hline Crystal system & Triclinic & \\
\hline Space group & $P 1$ & \\
\hline \multirow[t]{3}{*}{ Unit cell dimensions } & $a=9.7828(9) \AA$ & $\alpha=78.978(2)^{\circ}$ \\
\hline & $b=9.8015(9) \AA$ & $\beta=82.309(2)^{\circ}$ \\
\hline & $c=15.2502(14) \AA$ & $\gamma=68.047(2)^{\circ}$ \\
\hline Volume & $1336.8(2) \AA^{3}$ & \\
\hline$Z$ & 2 & \\
\hline Density (calculated) & $1.209 \mathrm{Mg} / \mathrm{m}^{3}$ & \\
\hline Absorption coefficient & $0.081 \mathrm{~mm}^{-1}$ & \\
\hline$F(000)$ & 520 & \\
\hline Crystal size & $0.34 \times 0.31 \times 0.15 \mathrm{~mm}^{3}$ & \\
\hline$\Theta$ range for data collection & 2.27 to $24.73^{\circ}$ & \\
\hline Index ranges & $-11 \leq h \leq 7,-11 \leq k \leq 9,18 \leq \ell \leq 18$ & \\
\hline Reflections collected & 6974 & \\
\hline Independent reflections & $4471\left[R_{\mathrm{int}}=0.0274\right]$ & \\
\hline Completeness to $\Theta=24.73^{\circ}$ & $97.6 \%$ & \\
\hline Absorption correction & None & \\
\hline Refinement method & Full-matrix least-squares on $F^{2}$ & \\
\hline Data / restraints / parameters & 4471 / 0 / 334 & \\
\hline Goodness-of-fit on $F^{2}$ & 1.021 & \\
\hline Final $R$ indices $[\mathrm{I}>2 \sigma(I)]$ & $R_{1}=0.0720, w R_{2}=0.1568$ & \\
\hline$R$ indices (all data) & $R_{1}=0.1084, w R_{2}=0.1738$ & \\
\hline Largest diff. peak and hole & 0.476 and $-0.284 \mathrm{e} / \AA^{3}$ & \\
\hline
\end{tabular}


Tables S.1.2. Atomic coordinates $\left(\times 10^{4}\right)$ and equivalent isotropic displacement parameters $\left(\AA^{2} \times 10^{3}\right)$ for 03084a. U(eq) is defined as one third of the trace of the orthogonalized $U_{i j}$ tensor.

\begin{tabular}{|c|c|c|c|c|}
\hline Atom & $\mathrm{x}$ & $\mathrm{y}$ & $\mathrm{z}$ & $\mathrm{U}(\mathrm{eq})$ \\
\hline$C(1)$ & 2435(3) & 7977(3) & $2723(2)$ & 23(1) \\
\hline$C(2)$ & $1525(3)$ & 9389(3) & 2921(2) & $25(1)$ \\
\hline$C(3)$ & 1189(3) & 10581(3) & $2233(2)$ & $28(1)$ \\
\hline$C(4)$ & 1697(4) & $10418(3)$ & $1362(2)$ & $28(1)$ \\
\hline $\mathrm{C}(5)$ & 2612(4) & $9002(3)$ & 1201(2) & $30(1)$ \\
\hline $\mathrm{C}(6)$ & $3007(3)$ & 7762(3) & $1868(2)$ & $25(1)$ \\
\hline$C(7)$ & $4036(3)$ & $6234(3)$ & $1647(2)$ & $28(1)$ \\
\hline$C(8)$ & 4104(3) & 5033(3) & $2457(2)$ & $26(1)$ \\
\hline $\mathrm{C}(9)$ & $4810(3)$ & $3530(3)$ & $2388(2)$ & $29(1)$ \\
\hline $\mathrm{C}(10)$ & $4876(3)$ & 2397(3) & $3098(2)$ & $28(1)$ \\
\hline $\mathrm{C}(11)$ & 4176(3) & 2826(3) & $3907(2)$ & $29(1)$ \\
\hline$C(12)$ & $3481(3)$ & 4309(3) & $4019(2)$ & $26(1)$ \\
\hline$C(13)$ & $3476(3)$ & $5397(3)$ & $3282(2)$ & $25(1)$ \\
\hline $\mathrm{C}(14)$ & $1276(4)$ & $11770(4)$ & $622(2)$ & $39(1)$ \\
\hline$C(15)$ & $-408(5)$ & $12490(4)$ & $636(3)$ & $56(1)$ \\
\hline$C(16)$ & 1904(5) & $12911(4)$ & $787(3)$ & $51(1)$ \\
\hline $\mathrm{C}(17)$ & $1875(6)$ & $11353(4)$ & $-301(2)$ & $66(1)$ \\
\hline $\mathrm{C}(18)$ & $3445(4)$ & $5868(4)$ & $878(2)$ & $41(1)$ \\
\hline$C(19)$ & $5588(4)$ & $6283(4)$ & 1383(3) & $45(1)$ \\
\hline$C(20)$ & $5717(4)$ & $752(3)$ & $3001(2)$ & $36(1)$ \\
\hline$C(21)$ & $4762(12)$ & $-169(10)$ & $3414(7)$ & $50(3)$ \\
\hline$C(22)$ & $7055(10)$ & 203(10) & $3648(6)$ & $56(3)$ \\
\hline$C(23)$ & $6347(13)$ & $473(12)$ & $2129(7)$ & 73(3) \\
\hline$C(21 \mathrm{~A})$ & $5400(10)$ & $-331(9)$ & $3743(6)$ & $57(2)$ \\
\hline$C(22 \mathrm{~A})$ & 7343(9) & $470(9)$ & $2800(6)$ & $63(3)$ \\
\hline$C(23 \mathrm{~A})$ & $5230(8)$ & $426(8)$ & $2126(5)$ & $46(2)$ \\
\hline $\mathrm{C}(24)$ & 2788(3) & 4703(3) & $4909(2)$ & $29(1)$ \\
\hline$C(25)$ & $3138(4)$ & $5700(4)$ & $5314(2)$ & $33(1)$ \\
\hline$C(26)$ & $2585(4)$ & 5994(4) & $6165(2)$ & $39(1)$ \\
\hline$C(27)$ & $1655(4)$ & $5332(4)$ & $6619(2)$ & $34(1)$ \\
\hline
\end{tabular}




\begin{tabular}{lrrrr}
$\mathrm{C}(28)$ & $1230(3)$ & $4364(3)$ & $6254(2)$ & $31(1)$ \\
$\mathrm{C}(29)$ & $1829(3)$ & $4056(3)$ & $5388(2)$ & $32(1)$ \\
$\mathrm{C}(30)$ & $233(4)$ & $3667(4)$ & $6728(3)$ & $43(1)$ \\
$\mathrm{C}(31)$ & $881(3)$ & $9638(3)$ & $3836(2)$ & $26(1)$ \\
$\mathrm{O}(1)$ & $2760(2)$ & $6847(2)$ & $3438(1)$ & $29(1)$ \\
$\mathrm{O}(2)$ & $540(3)$ & $8715(2)$ & $4377(1)$ & $37(1)$ \\
$\mathrm{O}(3)$ & $677(3)$ & $10965(2)$ & $4002(2)$ & $42(1)$ \\
$\mathrm{O}(4)$ & $1103(3)$ & $5645(3)$ & $7462(2)$ & $49(1)$ \\
$\mathrm{O}(5)$ & $-278(3)$ & $3828(3)$ & $7496(2)$ & $55(1)$ \\
\hline
\end{tabular}


Table S1.3. Bond lengths ( $\AA$ ) for 2,7-di-tert-butyl-5-(3-formyl-4-hydroxyphenyl)-9,9-dimethyl9H-xanthene-4-carboxylic acid (9).

\begin{tabular}{llll}
\hline Bond & Length & Bond & Length \\
\hline $\mathrm{C}(1)-\mathrm{O}(1)$ & $1.377(3)$ & $\mathrm{C}(13)-\mathrm{O}(1)$ & $1.382(3)$ \\
$\mathrm{C}(1)-\mathrm{C}(6)$ & $1.380(4)$ & $\mathrm{C}(14)-\mathrm{C}(17)$ & $1.529(5)$ \\
$\mathrm{C}(1)-\mathrm{C}(2)$ & $1.402(4)$ & $\mathrm{C}(14)-\mathrm{C}(15)$ & $1.529(5)$ \\
$\mathrm{C}(2)-\mathrm{C}(3)$ & $1.387(4)$ & $\mathrm{C}(14)-\mathrm{C}(16)$ & $1.535(5)$ \\
$\mathrm{C}(2)-\mathrm{C}(31)$ & $1.487(4)$ & $\mathrm{C}(20)-\mathrm{C}(23)$ & $1.427(11)$ \\
$\mathrm{C}(3)-\mathrm{C}(4)$ & $1.380(4)$ & $\mathrm{C}(20)-\mathrm{C}(21 \mathrm{~A})$ & $1.486(8)$ \\
$\mathrm{C}(4)-\mathrm{C}(5)$ & $1.390(4)$ & $\mathrm{C}(20)-\mathrm{C}(22 \mathrm{~A})$ & $1.508(8)$ \\
$\mathrm{C}(4)-\mathrm{C}(14)$ & $1.536(4)$ & $\mathrm{C}(20)-\mathrm{C}(21)$ & $1.531(9)$ \\
$\mathrm{C}(5)-\mathrm{C}(6)$ & $1.397(4)$ & $\mathrm{C}(20)-\mathrm{C}(23 \mathrm{~A})$ & $1.607(8)$ \\
$\mathrm{C}(6)-\mathrm{C}(7)$ & $1.531(4)$ & $\mathrm{C}(20)-\mathrm{C}(22)$ & $1.614(10)$ \\
$\mathrm{C}(7)-\mathrm{C}(8)$ & $1.529(4)$ & $\mathrm{C}(24)-\mathrm{C}(29)$ & $1.379(4)$ \\
$\mathrm{C}(7)-\mathrm{C}(19)$ & $1.534(5)$ & $\mathrm{C}(24)-\mathrm{C}(25)$ & $1.413(4)$ \\
$\mathrm{C}(7)-\mathrm{C}(18)$ & $1.535(5)$ & $\mathrm{C}(25)-\mathrm{C}(26)$ & $1.386(5)$ \\
$\mathrm{C}(8)-\mathrm{C}(13)$ & $1.382(4)$ & $\mathrm{C}(26)-\mathrm{C}(27)$ & $1.354(5)$ \\
$\mathrm{C}(8)-\mathrm{C}(9)$ & $1.392(4)$ & $\mathrm{C}(27)-\mathrm{O}(4)$ & $1.379(4)$ \\
$\mathrm{C}(9)-\mathrm{C}(10)$ & $1.389(4)$ & $\mathrm{C}(27)-\mathrm{C}(28)$ & $1.396(5)$ \\
$\mathrm{C}(10)-\mathrm{C}(11)$ & $1.393(4)$ & $\mathrm{C}(28)-\mathrm{C}(29)$ & $1.419(4)$ \\
$\mathrm{C}(10)-\mathrm{C}(20)$ & $1.539(4)$ & $\mathrm{C}(28)-\mathrm{C}(30)$ & $1.437(5)$ \\
$\mathrm{C}(11)-\mathrm{C}(12)$ & $1.389(4)$ & $\mathrm{C}(30)-\mathrm{O}(5)$ & $1.233(4)$ \\
$\mathrm{C}(12)-\mathrm{C}(13)$ & $1.397(4)$ & $\mathrm{C}(31)-\mathrm{O}(2)$ & $1.218(4)$ \\
$\mathrm{C}(12)-\mathrm{C}(24)$ & $1.495(4)$ & $\mathrm{C}(31)-\mathrm{O}(3)$ & $1.310(4)$ \\
\hline & & & \\
\hline
\end{tabular}


Table S1.4. Bond angles $\left[^{\circ}\right]$ for 2,7-di-tert-butyl-5-(3-formyl-4-hydroxyphenyl)-9,9dimethyl-9H-xanthene-4-carboxylic acid (9).

\begin{tabular}{|c|c|c|c|}
\hline Bond Angle & Angle & Bond Angle & Angle \\
\hline $\mathrm{O}(1)-\mathrm{C}(1)-\mathrm{C}(6)$ & $122.6(3)$ & $\mathrm{O}(1)-\mathrm{C}(13)-\mathrm{C}(8)$ & $123.1(3)$ \\
\hline $\mathrm{O}(1)-\mathrm{C}(1)-\mathrm{C}(2)$ & $115.9(3)$ & $\mathrm{O}(1)-\mathrm{C}(13)-\mathrm{C}(12)$ & $114.9(3)$ \\
\hline$C(6)-C(1)-C(2)$ & $121.4(3)$ & $C(8)-C(13)-C(12)$ & $122.0(3)$ \\
\hline$C(3)-C(2)-C(1)$ & $118.6(3)$ & $C(17)-C(14)-C(15)$ & $108.9(3)$ \\
\hline$C(3)-C(2)-C(31)$ & 119.2(3) & $C(17)-C(14)-C(16)$ & $108.0(3)$ \\
\hline$C(1)-C(2)-C(31)$ & $122.2(3)$ & $C(15)-C(14)-C(16)$ & $108.9(3)$ \\
\hline$C(4)-C(3)-C(2)$ & $122.2(3)$ & $C(17)-C(14)-C(4)$ & $112.4(3)$ \\
\hline$C(3)-C(4)-C(5)$ & $117.0(3)$ & $C(15)-C(14)-C(4)$ & $109.0(3)$ \\
\hline$C(3)-C(4)-C(14)$ & $120.2(3)$ & $C(16)-C(14)-C(4)$ & $109.5(3)$ \\
\hline$C(5)-C(4)-C(14)$ & $122.8(3)$ & $C(23)-C(20)-C(21 A)$ & $126.9(6)$ \\
\hline$C(4)-C(5)-C(6)$ & $123.4(3)$ & $\mathrm{C}(23)-\mathrm{C}(20)-\mathrm{C}(22 \mathrm{~A})$ & $61.8(6)$ \\
\hline$C(1)-C(6)-C(5)$ & 117.3(3) & $C(21 A)-C(20)-C(22 A)$ & $113.5(5)$ \\
\hline$C(1)-C(6)-C(7)$ & $122.0(3)$ & $\mathrm{C}(23)-\mathrm{C}(20)-\mathrm{C}(21)$ & $113.5(6)$ \\
\hline$C(5)-C(6)-C(7)$ & $120.8(3)$ & $C(21 \mathrm{~A})-\mathrm{C}(20)-\mathrm{C}(21)$ & $30.9(4)$ \\
\hline$C(8)-C(7)-C(6)$ & $110.3(2)$ & $\mathrm{C}(22 \mathrm{~A})-\mathrm{C}(20)-\mathrm{C}(21)$ & $136.6(5)$ \\
\hline$C(8)-C(7)-C(19)$ & $109.3(3)$ & $C(23)-C(20)-C(10)$ & $115.7(5)$ \\
\hline$C(6)-C(7)-C(19)$ & $108.7(3)$ & $C(21 A)-C(20)-C(10)$ & $114.8(4)$ \\
\hline$C(8)-C(7)-C(18)$ & $109.0(3)$ & $\mathrm{C}(22 \mathrm{~A})-\mathrm{C}(20)-\mathrm{C}(10)$ & $110.0(4)$ \\
\hline$C(6)-C(7)-C(18)$ & $109.2(3)$ & $\mathrm{C}(21)-\mathrm{C}(20)-\mathrm{C}(10)$ & $109.8(4)$ \\
\hline $\mathrm{C}(19)-\mathrm{C}(7)-\mathrm{C}(18)$ & $110.4(3)$ & $C(23)-C(20)-C(23 A)$ & $42.4(5)$ \\
\hline $\mathrm{C}(13)-\mathrm{C}(8)-\mathrm{C}(9)$ & $117.6(3)$ & $C(21 A)-C(20)-C(23 A)$ & $105.3(5)$ \\
\hline$C(13)-C(8)-C(7)$ & $121.4(3)$ & $C(22 A)-C(20)-C(23 A)$ & $103.7(5)$ \\
\hline $\mathrm{C}(9)-\mathrm{C}(8)-\mathrm{C}(7)$ & $121.0(3)$ & $C(21)-C(20)-C(23 A)$ & $78.9(5)$ \\
\hline $\mathrm{C}(10)-\mathrm{C}(9)-\mathrm{C}(8)$ & $123.2(3)$ & $C(10)-C(20)-C(23 A)$ & $108.8(3)$ \\
\hline $\mathrm{C}(9)-\mathrm{C}(10)-\mathrm{C}(11)$ & $116.8(3)$ & $C(23)-C(20)-C(22)$ & $107.7(6)$ \\
\hline C(9)-C(10)-C(20) & $121.4(3)$ & $C(21 A)-C(20)-C(22)$ & $73.6(5)$ \\
\hline$C(11)-C(10)-C(20)$ & $121.8(3)$ & $C(22 A)-C(20)-C(22)$ & $48.7(4)$ \\
\hline$C(12)-C(11)-C(10)$ & $122.6(3)$ & $\mathrm{C}(21)-\mathrm{C}(20)-\mathrm{C}(22)$ & $104.3(6)$ \\
\hline$C(11)-C(12)-C(13)$ & $117.9(3)$ & $C(10)-C(20)-C(22)$ & $104.8(4)$ \\
\hline$C(11)-C(12)-C(24)$ & $120.2(3)$ & $C(23 \mathrm{~A})-\mathrm{C}(20)-\mathrm{C}(22)$ & $142.8(5)$ \\
\hline$C(13)-C(12)-C(24)$ & $121.9(3)$ & $\mathrm{C}(29)-\mathrm{C}(24)-\mathrm{C}(25)$ & $117.2(3)$ \\
\hline
\end{tabular}




\begin{tabular}{llll}
$\mathrm{O}(1)-\mathrm{C}(1)-\mathrm{C}(6)$ & $122.6(3)$ & $\mathrm{O}(1)-\mathrm{C}(13)-\mathrm{C}(8)$ & $123.1(3)$ \\
$\mathrm{O}(1)-\mathrm{C}(1)-\mathrm{C}(2)$ & $115.9(3)$ & $\mathrm{O}(1)-\mathrm{C}(13)-\mathrm{C}(12)$ & $114.9(3)$ \\
$\mathrm{C}(29)-\mathrm{C}(24)-\mathrm{C}(12)$ & $121.3(3)$ & $\mathrm{C}(27)-\mathrm{C}(28)-\mathrm{C}(30)$ & $122.3(3)$ \\
$\mathrm{C}(25)-\mathrm{C}(24)-\mathrm{C}(12)$ & $121.4(3)$ & $\mathrm{C}(29)-\mathrm{C}(28)-\mathrm{C}(30)$ & $119.7(3)$ \\
$\mathrm{C}(26)-\mathrm{C}(25)-\mathrm{C}(24)$ & $122.1(3)$ & $\mathrm{C}(24)-\mathrm{C}(29)-\mathrm{C}(28)$ & $121.5(3)$ \\
$\mathrm{C}(27)-\mathrm{C}(26)-\mathrm{C}(25)$ & $119.2(3)$ & $\mathrm{O}(5)-\mathrm{C}(30)-\mathrm{C}(28)$ & $123.4(4)$ \\
$\mathrm{C}(26)-\mathrm{C}(27)-\mathrm{O}(4)$ & $118.6(3)$ & $\mathrm{O}(2)-\mathrm{C}(31)-\mathrm{O}(3)$ & $122.8(3)$ \\
$\mathrm{C}(26)-\mathrm{C}(27)-\mathrm{C}(28)$ & $121.9(3)$ & $\mathrm{O}(2)-\mathrm{C}(31)-\mathrm{C}(2)$ & $123.6(3)$ \\
$\mathrm{O}(4)-\mathrm{C}(27)-\mathrm{C}(28)$ & $119.5(3)$ & $\mathrm{O}(3)-\mathrm{C}(31)-\mathrm{C}(2)$ & $113.6(3)$ \\
$\mathrm{C}(27)-\mathrm{C}(28)-\mathrm{C}(29)$ & $118.0(3)$ & $\mathrm{C}(1)-\mathrm{O}(1)-\mathrm{C}(13)$ & $119.0(2)$ \\
\hline
\end{tabular}


Table S1.5. Anisotropic displacement parameters $\left(\AA^{2} \times 10^{3}\right)$ for 2,7-di-tert-butyl-5-(3formyl-4-hydroxyphenyl)-9,9-dimethyl-9H-xanthene-4-carboxylic acid (9). The anisotropic displacement factor exponent takes the form: $-2 \pi^{2}\left[h^{2} a^{* 2} U_{11}+\ldots+\right.$ $\left.2 \mathrm{hka} * \mathrm{~b} * \mathrm{U}_{12}\right]$.

\begin{tabular}{|c|c|c|c|c|c|c|}
\hline Atom & $\mathrm{U}_{11}$ & $\mathrm{U}_{22}$ & $\mathrm{U}_{33}$ & $\mathrm{U}_{23}$ & $\mathrm{U}_{13}$ & $\mathrm{U}_{12}$ \\
\hline$C(1)$ & $22(2)$ & $19(2)$ & $25(2)$ & $1(1)$ & $-2(1)$ & $-5(1)$ \\
\hline$C(2)$ & $23(2)$ & $24(2)$ & $27(2)$ & $-2(1)$ & $-1(1)$ & $-7(1)$ \\
\hline$C(3)$ & $31(2)$ & $20(2)$ & $31(2)$ & $-6(1)$ & $-1(1)$ & $-5(1)$ \\
\hline$C(4)$ & $34(2)$ & $19(2)$ & $29(2)$ & $-1(1)$ & $-4(1)$ & $-9(1)$ \\
\hline$C(5)$ & $40(2)$ & $27(2)$ & $21(2)$ & $-3(1)$ & $4(1)$ & $-13(2)$ \\
\hline$C(6)$ & $26(2)$ & $24(2)$ & $24(2)$ & $-5(1)$ & $2(1)$ & $-9(1)$ \\
\hline$C(7)$ & $31(2)$ & $23(2)$ & $25(2)$ & $-5(1)$ & $4(1)$ & $-5(1)$ \\
\hline$C(8)$ & $25(2)$ & $26(2)$ & $25(2)$ & $-5(1)$ & $0(1)$ & $-7(1)$ \\
\hline$C(9)$ & $28(2)$ & $27(2)$ & $27(2)$ & $-8(1)$ & $1(1)$ & $-4(1)$ \\
\hline$C(10)$ & $27(2)$ & $22(2)$ & $31(2)$ & $-1(1)$ & $-5(1)$ & $-4(1)$ \\
\hline $\mathrm{C}(11)$ & $30(2)$ & $24(2)$ & $28(2)$ & $3(1)$ & $-1(1)$ & $-7(1)$ \\
\hline$C(12)$ & $23(2)$ & $23(2)$ & $27(2)$ & $-3(1)$ & $-3(1)$ & $-3(1)$ \\
\hline$C(13)$ & $21(2)$ & $21(2)$ & $28(2)$ & $-5(1)$ & $-2(1)$ & $-3(1)$ \\
\hline$C(14)$ & $55(2)$ & $23(2)$ & $31(2)$ & $2(2)$ & $-1(2)$ & $-9(2)$ \\
\hline$C(15)$ & $66(3)$ & $38(2)$ & $57(3)$ & $10(2)$ & $-25(2)$ & $-13(2)$ \\
\hline$C(16)$ & $74(3)$ & $33(2)$ & $47(2)$ & $1(2)$ & $4(2)$ & $-26(2)$ \\
\hline$C(17)$ & $122(4)$ & $38(2)$ & $29(2)$ & $4(2)$ & $-3(2)$ & $-22(2)$ \\
\hline$C(18)$ & $62(3)$ & $28(2)$ & $28(2)$ & $-5(2)$ & $-8(2)$ & $-8(2)$ \\
\hline$C(19)$ & $39(2)$ & $34(2)$ & $52(2)$ & $-1(2)$ & $14(2)$ & $-9(2)$ \\
\hline$C(20)$ & $38(2)$ & $20(2)$ & $42(2)$ & $-6(2)$ & $2(2)$ & $-3(2)$ \\
\hline$C(24)$ & $26(2)$ & $22(2)$ & $30(2)$ & $4(1)$ & $-3(1)$ & $0(1)$ \\
\hline$C(25)$ & $34(2)$ & $30(2)$ & $32(2)$ & $-5(2)$ & $-1(2)$ & $-9(2)$ \\
\hline$C(26)$ & $42(2)$ & $35(2)$ & $39(2)$ & $-6(2)$ & $-8(2)$ & $-12(2)$ \\
\hline $\mathrm{C}(27)$ & $33(2)$ & $27(2)$ & $34(2)$ & $-3(2)$ & $-5(2)$ & $-2(2)$ \\
\hline$C(28)$ & $27(2)$ & $23(2)$ & $37(2)$ & $2(1)$ & $-3(2)$ & $-5(1)$ \\
\hline C(29) & $31(2)$ & $25(2)$ & $33(2)$ & $-3(1)$ & $-3(2)$ & $-4(1)$ \\
\hline$C(30)$ & $34(2)$ & $39(2)$ & $52(3)$ & $-5(2)$ & $1(2)$ & $-10(2)$ \\
\hline $\mathrm{C}(31)$ & $27(2)$ & $18(2)$ & $28(2)$ & $-2(1)$ & $-1(1)$ & $-3(1)$ \\
\hline $\mathrm{O}(1)$ & $35(1)$ & $19(1)$ & $23(1)$ & $-2(1)$ & $0(1)$ & $0(1)$ \\
\hline
\end{tabular}




\begin{tabular}{llllrrr}
$\mathrm{O}(2)$ & $52(2)$ & $24(1)$ & $31(1)$ & $-8(1)$ & $11(1)$ & $-11(1)$ \\
$\mathrm{O}(3)$ & $69(2)$ & $25(1)$ & $32(1)$ & $-12(1)$ & $16(1)$ & $-18(1)$ \\
$\mathrm{O}(4)$ & $57(2)$ & $55(2)$ & $32(1)$ & $-14(1)$ & $8(1)$ & $-18(1)$ \\
$\mathrm{O}(5)$ & $59(2)$ & $60(2)$ & $42(2)$ & $-11(1)$ & $19(1)$ & $-22(1)$ \\
\hline
\end{tabular}


Table S1.6. Hydrogen coordinates $\left(\times 10^{4}\right)$ and isotropic displacement parameters $\left(\AA^{2} \times 10^{3}\right)$ for 2,7-di-tert-butyl-5-(3-formyl-4-hydroxyphenyl)-9,9-dimethyl-9H-xanthene-4-carboxylic acid (9).

\begin{tabular}{|c|c|c|c|c|}
\hline Atom & $\mathrm{x}$ & $\mathrm{y}$ & $\mathrm{z}$ & $\mathrm{U}(\mathrm{eq})$ \\
\hline $\mathrm{H}(3)$ & 602 & 11522 & 2362 & 34 \\
\hline $\mathrm{H}(5)$ & 2981 & 8872 & 620 & 36 \\
\hline $\mathrm{H}(9)$ & 5260 & 3272 & 1840 & 34 \\
\hline $\mathrm{H}(11)$ & 4174 & 2091 & 4389 & 35 \\
\hline $\mathrm{H}(15 \mathrm{~A})$ & -680 & 13322 & 165 & 84 \\
\hline $\mathrm{H}(15 \mathrm{~B})$ & -788 & 12827 & 1198 & 84 \\
\hline $\mathrm{H}(15 \mathrm{C})$ & -813 & 11773 & 555 & 84 \\
\hline $\mathrm{H}(16 \mathrm{~A})$ & 2962 & 12473 & 771 & 76 \\
\hline $\mathrm{H}(16 \mathrm{~B})$ & 1527 & 13204 & 1360 & 76 \\
\hline $\mathrm{H}(16 \mathrm{C})$ & 1616 & 13769 & 334 & 76 \\
\hline $\mathrm{H}(17 \mathrm{~A})$ & 1474 & 10659 & -425 & 99 \\
\hline $\mathrm{H}(17 \mathrm{~B})$ & 2933 & 10905 & -317 & 99 \\
\hline $\mathrm{H}(17 \mathrm{C})$ & 1594 & 12231 & -739 & 99 \\
\hline $\mathrm{H}(18 \mathrm{~A})$ & 4055 & 4888 & 760 & 62 \\
\hline $\mathrm{H}(18 \mathrm{~B})$ & 3461 & 6581 & 356 & 62 \\
\hline $\mathrm{H}(18 \mathrm{C})$ & 2450 & 5908 & 1038 & 62 \\
\hline $\mathrm{H}(19 \mathrm{~A})$ & 5928 & 6550 & 1861 & 68 \\
\hline $\mathrm{H}(19 \mathrm{~B})$ & 5556 & 7007 & 860 & 68 \\
\hline $\mathrm{H}(19 \mathrm{C})$ & 6251 & 5321 & 1262 & 68 \\
\hline $\mathrm{H}(21 \mathrm{~A})$ & 3894 & 147 & 3090 & 75 \\
\hline $\mathrm{H}(21 \mathrm{~B})$ & 4477 & -28 & 4023 & 75 \\
\hline $\mathrm{H}(21 \mathrm{C})$ & 5316 & -1203 & 3388 & 75 \\
\hline $\mathrm{H}(22 \mathrm{~A})$ & 7580 & -847 & 3662 & 84 \\
\hline $\mathrm{H}(22 \mathrm{~B})$ & 6664 & 400 & 4237 & 84 \\
\hline $\mathrm{H}(22 \mathrm{C})$ & 7716 & 727 & 3428 & 84 \\
\hline $\mathrm{H}(23 \mathrm{~A})$ & 6720 & -580 & 2116 & 109 \\
\hline $\mathrm{H}(23 \mathrm{~B})$ & 7140 & 846 & 1977 & 109 \\
\hline $\mathrm{H}(23 \mathrm{C})$ & 5604 & 964 & 1709 & 109 \\
\hline $\mathrm{H}(21 \mathrm{D})$ & 4351 & -90 & 3840 & 86 \\
\hline $\mathrm{H}(21 \mathrm{E})$ & 5788 & -285 & 4274 & 86 \\
\hline
\end{tabular}




\begin{tabular}{lrrrl}
$\mathrm{H}(21 \mathrm{~F})$ & 5855 & -1317 & 3593 & 86 \\
$\mathrm{H}(22 \mathrm{D})$ & 7851 & -544 & 2706 & 95 \\
$\mathrm{H}(22 \mathrm{E})$ & 7732 & 647 & 3292 & 95 \\
$\mathrm{H}(22 \mathrm{~F})$ & 7482 & 1127 & 2275 & 95 \\
$\mathrm{H}(23 \mathrm{D})$ & 5465 & 1052 & 1613 & 69 \\
$\mathrm{H}(23 \mathrm{E})$ & 4186 & 629 & 2182 & 69 \\
$\mathrm{H}(23 \mathrm{~F})$ & 5749 & -599 & 2059 & 69 \\
$\mathrm{H}(25)$ & 3760 & 6173 & 4999 & 39 \\
$\mathrm{H}(26)$ & 2850 & 6638 & 6421 & 47 \\
$\mathrm{H}(29)$ & 1569 & 3403 & 5137 & 38 \\
$\mathrm{H}(30)$ & -41 & 3064 & 6441 & 52 \\
$\mathrm{H}(3 \mathrm{~A})$ & 322 & 11042 & 4513 & 64 \\
$\mathrm{H}(4)$ & 554 & 5185 & 7666 & 73 \\
\hline
\end{tabular}




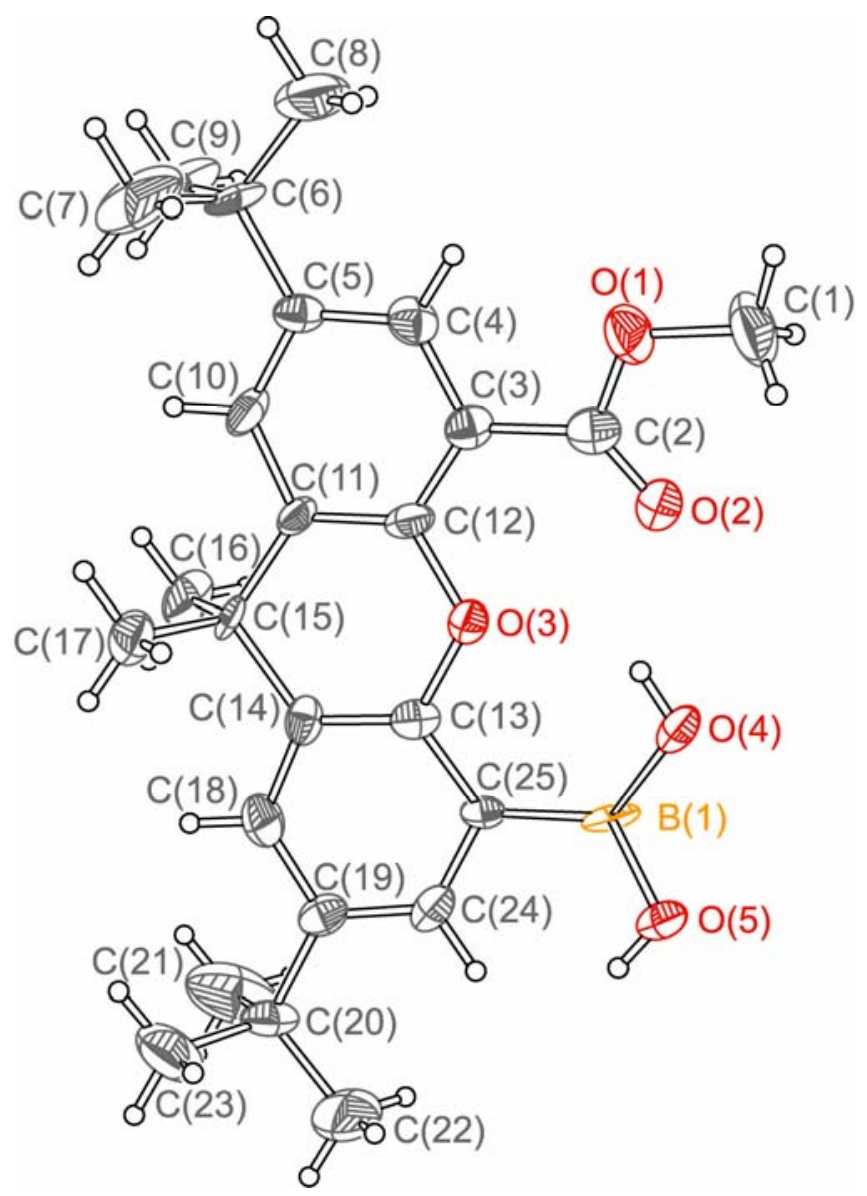

Figure S2. Thermal ellipsoid plot of 5-(boronic acid)-2,7-di-tert-butyl-9,9-dimethyl-9Hxanthene-4-carboxylic acid methyl ester (24). Thermal ellipsoids are drawn at the 50\% probability level. 
Table S2.1. Crystal data and structure refinement for 5-(boronic acid)-2,7-di-tert-butyl9,9-dimethyl-9H-xanthene-4-carboxylic acid methyl ester (24).

\begin{tabular}{|c|c|c|}
\hline Identification code & 030780a & \\
\hline Empirical formula & C25 H33 BO5 & \\
\hline Formula weight & 424.32 & \\
\hline Temperature & $467(2) \mathrm{K}$ & \\
\hline Wavelength & $0.71073 \AA$ & \\
\hline Crystal system & Triclinic & \\
\hline Space group & $P \overline{1}$ & \\
\hline \multirow[t]{3}{*}{ Unit cell dimensions } & $a=9.157(7) \AA$ & $\alpha=77.035(14)^{\circ}$ \\
\hline & $b=11.143(10) \AA$ & $\beta=83.053(17)^{\circ}$ \\
\hline & $c=12.500(11) \AA$ & $\gamma=79.421(18)^{\circ}$ \\
\hline Volume & $1217.6(18) \AA^{3}$ & \\
\hline$Z$ & 2 & \\
\hline Density (calculated) & $1.157 \mathrm{Mg} / \mathrm{m}^{3}$ & \\
\hline Absorption coefficient & $0.078 \mathrm{~mm}^{-1}$ & \\
\hline$F(000)$ & 456 & \\
\hline Crystal size & $0.20 \times 0.17 \times 0.04 \mathrm{~mm}^{3}$ & \\
\hline$\Theta$ range for data collection & 2.26 to $24.69^{\circ}$ & \\
\hline Index ranges & $-8 \leq h \leq 7,-11 \leq k \leq 12,-5 \leq \ell \leq 14$ & \\
\hline Reflections collected & 2658 & \\
\hline Independent reflections & $2654\left[R_{\mathrm{int}}=0.0508\right]$ & \\
\hline Completeness to $\Theta=24.69^{\circ}$ & $63.9 \%$ & \\
\hline Absorption correction & None & \\
\hline Refinement method & Full-matrix least-squares on $F^{2}$ & \\
\hline Data / restraints / parameters & 2654 / 0 / 291 & \\
\hline Goodness-of-fit on $F^{2}$ & 1.083 & \\
\hline Final $R$ indices $[\mathrm{I}>2 \sigma(I)]$ & $R_{1}=0.0970, w R_{2}=0.1710$ & \\
\hline$R$ indices (all data) & $R_{1}=0.1935, w R_{2}=0.2057$ & \\
\hline Largest diff. peak and hole & 0.201 and $-0.212 \mathrm{e} / \AA^{3}$ & \\
\hline
\end{tabular}


Tables S2.2. Atomic coordinates $\left(\times 10^{4}\right)$ and equivalent isotropic displacement parameters $\left(\AA^{2} \times 10^{3}\right)$ for 5-(boronic acid)-2,7-di-tert-butyl-9,9-dimethyl-9H-xanthene-4carboxylic acid methyl ester (24). $\mathrm{U}(\mathrm{eq})$ is defined as one third of the trace of the orthogonalized $\mathrm{U}_{\mathrm{ij}}$ tensor.

\begin{tabular}{|c|c|c|c|c|}
\hline Atom & $\mathrm{x}$ & $\mathrm{y}$ & $\mathrm{z}$ & $\mathrm{U}(\mathrm{eq})$ \\
\hline $\mathrm{B}(1)$ & $13069(11)$ & $5899(8)$ & $5728(7)$ & $28(3)$ \\
\hline $\mathrm{O}(1)$ & $10585(5)$ & $5580(4)$ & 1694(4) & $49(2)$ \\
\hline $\mathrm{O}(2)$ & $11546(5)$ & $5096(4)$ & $3323(4)$ & $47(2)$ \\
\hline $\mathrm{O}(3)$ & $10351(5)$ & $6702(4)$ & 4621(3) & $31(1)$ \\
\hline $\mathrm{O}(4)$ & $13180(5)$ & $5266(5)$ & 4906(4) & $41(2)$ \\
\hline $\mathrm{O}(5)$ & $14354(5)$ & $5789(5)$ & $6219(4)$ & $45(2)$ \\
\hline $\mathrm{C}(1)$ & 11852(9) & $4767(7)$ & 1304(6) & $62(3)$ \\
\hline$C(2)$ & 10577(9) & $5675(6)$ & $2750(6)$ & $33(2)$ \\
\hline $\mathrm{C}(3)$ & $9208(8)$ & $6502(6)$ & $3104(5)$ & $29(2)$ \\
\hline $\mathrm{C}(4)$ & 7976(8) & $6787(6)$ & $2448(5)$ & $34(2)$ \\
\hline $\mathrm{C}(5)$ & $6676(7)$ & $7557(6)$ & $2734(5)$ & $29(2)$ \\
\hline $\mathrm{C}(6)$ & 5343(9) & 7909(7) & 2019(6) & $38(2)$ \\
\hline$C(7)$ & $5169(10)$ & $9299(7)$ & $1528(6)$ & $88(3)$ \\
\hline$C(8)$ & 5533(9) & 7184(9) & $1106(7)$ & $97(4)$ \\
\hline $\mathrm{C}(9)$ & 3913(8) & $7650(8)$ & $2735(6)$ & $65(3)$ \\
\hline $\mathrm{C}(10)$ & $6663(8)$ & $8017(6)$ & $3663(5)$ & $34(2)$ \\
\hline $\mathrm{C}(11)$ & 7830(7) & $7785(6)$ & $4335(5)$ & $27(2)$ \\
\hline$C(12)$ & $9098(8)$ & $6996(6)$ & $4026(5)$ & $30(2)$ \\
\hline$C(13)$ & $10349(8)$ & $7162(6)$ & $5572(5)$ & $27(2)$ \\
\hline$C(14)$ & $9137(7)$ & 7964(6) & $5928(5)$ & $27(2)$ \\
\hline$C(15)$ & 7698(7) & $8386(6)$ & $5333(5)$ & $31(2)$ \\
\hline$C(16)$ & $6373(7)$ & $8017(7)$ & $6123(5)$ & $50(3)$ \\
\hline $\mathrm{C}(17)$ & 7523(7) & $9826(5)$ & 4916(5) & $44(2)$ \\
\hline$C(18)$ & $9292(8)$ & $8383(6)$ & $6870(5)$ & $38(2)$ \\
\hline$C(19)$ & 10531(8) & $8038(6)$ & 7464(5) & $31(2)$ \\
\hline$C(20)$ & 10566(9) & $8540(7)$ & $8503(5)$ & $39(2)$ \\
\hline $\mathrm{C}(21)$ & $9319(11)$ & $8128(9)$ & $9350(6)$ & $93(3)$ \\
\hline$C(22)$ & $12056(10)$ & $8103(9)$ & $9019(7)$ & 93(4) \\
\hline$C(23)$ & $10328(10)$ & 9978(7) & 8199(6) & $77(3)$ \\
\hline$C(24)$ & $11694(8)$ & $7234(6)$ & $7047(5)$ & $32(2)$ \\
\hline$C(25)$ & 11651(8) & $6775(6)$ & $6108(5)$ & $28(2)$ \\
\hline
\end{tabular}


Table S2.3. Bond lengths ( $\AA$ ) for 5-(boronic acid)-2,7-di-tert-butyl-9,9-dimethyl-9Hxanthene-4-carboxylic acid methyl ester (24).

\begin{tabular}{llll}
\hline Bond & Length & Bond & Length \\
\hline $\mathrm{B}(1)-\mathrm{O}(4)$ & $1.356(8)$ & $\mathrm{C}(10)-\mathrm{C}(11)$ & $1.390(8)$ \\
$\mathrm{B}(1)-\mathrm{O}(5)$ & $1.365(9)$ & $\mathrm{C}(11)-\mathrm{C}(12)$ & $1.392(8)$ \\
$\mathrm{B}(1)-\mathrm{C}(25)$ & $1.568(10)$ & $\mathrm{C}(11)-\mathrm{C}(15)$ & $1.526(8)$ \\
$\mathrm{O}(1)-\mathrm{C}(2)$ & $1.346(7)$ & $\mathrm{C}(13)-\mathrm{C}(14)$ & $1.386(8)$ \\
$\mathrm{O}(1)-\mathrm{C}(1)$ & $1.442(7)$ & $\mathrm{C}(13)-\mathrm{C}(25)$ & $1.390(8)$ \\
$\mathrm{O}(2)-\mathrm{C}(2)$ & $1.206(7)$ & $\mathrm{C}(14)-\mathrm{C}(18)$ & $1.391(8)$ \\
$\mathrm{O}(3)-\mathrm{C}(12)$ & $1.391(7)$ & $\mathrm{C}(14)-\mathrm{C}(15)$ & $1.537(8)$ \\
$\mathrm{O}(3)-\mathrm{C}(13)$ & $1.396(7)$ & $\mathrm{C}(15)-\mathrm{C}(16)$ & $1.525(9)$ \\
$\mathrm{C}(2)-\mathrm{C}(3)$ & $1.497(9)$ & $\mathrm{C}(15)-\mathrm{C}(17)$ & $1.556(8)$ \\
$\mathrm{C}(3)-\mathrm{C}(12)$ & $1.371(8)$ & $\mathrm{C}(18)-\mathrm{C}(19)$ & $1.379(9)$ \\
$\mathrm{C}(3)-\mathrm{C}(4)$ & $1.422(8)$ & $\mathrm{C}(19)-\mathrm{C}(24)$ & $1.390(8)$ \\
$\mathrm{C}(4)-\mathrm{C}(5)$ & $1.393(8)$ & $\mathrm{C}(19)-\mathrm{C}(20)$ & $1.532(8)$ \\
$\mathrm{C}(5)-\mathrm{C}(10)$ & $1.369(8)$ & $\mathrm{C}(20)-\mathrm{C}(21)$ & $1.522(10)$ \\
$\mathrm{C}(5)-\mathrm{C}(6)$ & $1.541(9)$ & $\mathrm{C}(20)-\mathrm{C}(22)$ & $1.530(10)$ \\
$\mathrm{C}(6)-\mathrm{C}(8)$ & $1.516(9)$ & $\mathrm{C}(20)-\mathrm{C}(23)$ & $1.542(9)$ \\
$\mathrm{C}(6)-\mathrm{C}(7)$ & $1.519(10)$ & $\mathrm{C}(24)-\mathrm{C}(25)$ & $1.390(8)$ \\
$\mathrm{C}(6)-\mathrm{C}(9)$ & $1.529(9)$ & & \\
\hline
\end{tabular}


Table S2.4. Bond angles $\left({ }^{\circ}\right)$ for 5-(boronic acid)-2,7-di-tert-butyl-9,9-dimethyl-9Hxanthene-4-carboxylic acid methyl ester (24).

\begin{tabular}{llll}
\hline Bond Angle & Angle & Bond Angle & Angle \\
\hline $\mathrm{O}(4)-\mathrm{B}(1)-\mathrm{O}(5)$ & $115.2(7)$ & $\mathrm{O}(3)-\mathrm{C}(12)-\mathrm{C}(11)$ & $121.5(6)$ \\
$\mathrm{O}(4)-\mathrm{B}(1)-\mathrm{C}(25)$ & $126.2(8)$ & $\mathrm{C}(14)-\mathrm{C}(13)-\mathrm{C}(25)$ & $123.4(6)$ \\
$\mathrm{O}(5)-\mathrm{B}(1)-\mathrm{C}(25)$ & $118.5(7)$ & $\mathrm{C}(14)-\mathrm{C}(13)-\mathrm{O}(3)$ & $121.6(6)$ \\
$\mathrm{C}(2)-\mathrm{O}(1)-\mathrm{C}(1)$ & $114.9(5)$ & $\mathrm{C}(25)-\mathrm{C}(13)-\mathrm{O}(3)$ & $114.9(6)$ \\
$\mathrm{C}(12)-\mathrm{O}(3)-\mathrm{C}(13)$ & $120.5(5)$ & $\mathrm{C}(13)-\mathrm{C}(14)-\mathrm{C}(18)$ & $116.0(6)$ \\
$\mathrm{O}(2)-\mathrm{C}(2)-\mathrm{O}(1)$ & $121.8(6)$ & $\mathrm{C}(13)-\mathrm{C}(14)-\mathrm{C}(15)$ & $123.2(6)$ \\
$\mathrm{O}(2)-\mathrm{C}(2)-\mathrm{C}(3)$ & $126.3(6)$ & $\mathrm{C}(18)-\mathrm{C}(14)-\mathrm{C}(15)$ & $120.9(6)$ \\
$\mathrm{O}(1)-\mathrm{C}(2)-\mathrm{C}(3)$ & $111.8(6)$ & $\mathrm{C}(16)-\mathrm{C}(15)-\mathrm{C}(11)$ & $110.1(6)$ \\
$\mathrm{C}(12)-\mathrm{C}(3)-\mathrm{C}(4)$ & $118.9(6)$ & $\mathrm{C}(16)-\mathrm{C}(15)-\mathrm{C}(14)$ & $109.7(5)$ \\
$\mathrm{C}(12)-\mathrm{C}(3)-\mathrm{C}(2)$ & $122.9(7)$ & $\mathrm{C}(11)-\mathrm{C}(15)-\mathrm{C}(14)$ & $109.8(5)$ \\
$\mathrm{C}(4)-\mathrm{C}(3)-\mathrm{C}(2)$ & $118.2(6)$ & $\mathrm{C}(16)-\mathrm{C}(15)-\mathrm{C}(17)$ & $111.4(5)$ \\
$\mathrm{C}(5)-\mathrm{C}(4)-\mathrm{C}(3)$ & $120.7(6)$ & $\mathrm{C}(11)-\mathrm{C}(15)-\mathrm{C}(17)$ & $108.2(5)$ \\
$\mathrm{C}(10)-\mathrm{C}(5)-\mathrm{C}(4)$ & $116.6(6)$ & $\mathrm{C}(14)-\mathrm{C}(15)-\mathrm{C}(17)$ & $107.7(6)$ \\
$\mathrm{C}(10)-\mathrm{C}(5)-\mathrm{C}(6)$ & $121.7(6)$ & $\mathrm{C}(19)-\mathrm{C}(18)-\mathrm{C}(14)$ & $124.7(6)$ \\
$\mathrm{C}(4)-\mathrm{C}(5)-\mathrm{C}(6)$ & $121.7(6)$ & $\mathrm{C}(18)-\mathrm{C}(19)-\mathrm{C}(24)$ & $115.5(6)$ \\
$\mathrm{C}(8)-\mathrm{C}(6)-\mathrm{C}(7)$ & $109.8(7)$ & $\mathrm{C}(18)-\mathrm{C}(19)-\mathrm{C}(20)$ & $120.2(6)$ \\
$\mathrm{C}(8)-\mathrm{C}(6)-\mathrm{C}(9)$ & $108.1(7)$ & $\mathrm{C}(24)-\mathrm{C}(19)-\mathrm{C}(20)$ & $124.3(6)$ \\
$\mathrm{C}(7)-\mathrm{C}(6)-\mathrm{C}(9)$ & $108.1(7)$ & $\mathrm{C}(21)-\mathrm{C}(20)-\mathrm{C}(22)$ & $109.1(7)$ \\
$\mathrm{C}(8)-\mathrm{C}(6)-\mathrm{C}(5)$ & $112.6(6)$ & $\mathrm{C}(21)-\mathrm{C}(20)-\mathrm{C}(19)$ & $109.7(7)$ \\
$\mathrm{C}(7)-\mathrm{C}(6)-\mathrm{C}(5)$ & $108.3(6)$ & $\mathrm{C}(22)-\mathrm{C}(20)-\mathrm{C}(19)$ & $112.6(6)$ \\
$\mathrm{C}(9)-\mathrm{C}(6)-\mathrm{C}(5)$ & $109.9(6)$ & $\mathrm{C}(21)-\mathrm{C}(20)-\mathrm{C}(23)$ & $109.0(7)$ \\
$\mathrm{C}(5)-\mathrm{C}(10)-\mathrm{C}(11)$ & $125.6(6)$ & $\mathrm{C}(22)-\mathrm{C}(20)-\mathrm{C}(23)$ & $107.5(7)$ \\
$\mathrm{C}(10)-\mathrm{C}(11)-\mathrm{C}(12)$ & $115.8(6)$ & $\mathrm{C}(19)-\mathrm{C}(20)-\mathrm{C}(23)$ & $108.9(6)$ \\
$\mathrm{C}(10)-\mathrm{C}(11)-\mathrm{C}(15)$ & $120.8(6)$ & $\mathrm{C}(19)-\mathrm{C}(24)-\mathrm{C}(25)$ & $124.0(6)$ \\
$\mathrm{C}(12)-\mathrm{C}(11)-\mathrm{C}(15)$ & $123.5(6)$ & $\mathrm{C}(13)-\mathrm{C}(25)-\mathrm{C}(24)$ & $116.4(6)$ \\
$\mathrm{C}(3)-\mathrm{C}(12)-\mathrm{O}(3)$ & $116.1(6)$ & $\mathrm{C}(13)-\mathrm{C}(25)-\mathrm{B}(1)$ & $125.7(6)$ \\
$\mathrm{C}(3)-\mathrm{C}(12)-\mathrm{C}(11)$ & $122.4(7)$ & $\mathrm{C}(24)-\mathrm{C}(25)-\mathrm{B}(1)$ & $117.9(7)$ \\
\hline & & & \\
& & & \\
& & &
\end{tabular}


Table S2.5. Anisotropic displacement parameters $\left(\AA^{2} \times 10^{3}\right)$ for 5-(boronic acid)-2,7-ditert-butyl-9,9-dimethyl-9H-xanthene-4-carboxylic acid methyl ester (24). The anisotropic displacement factor exponent takes the form: $-2 \pi^{2}\left[\mathrm{~h}^{2} \mathrm{a}^{* 2} \mathrm{U}_{11}+\ldots+2 \mathrm{hka} * \mathrm{~b}^{*} \mathrm{U}_{12}\right]$.

\begin{tabular}{|c|c|c|c|c|c|c|}
\hline Atom & $\mathrm{U}_{11}$ & $\mathrm{U}_{22}$ & $\mathrm{U}_{33}$ & $\mathrm{U}_{23}$ & $\mathrm{U}_{13}$ & $\mathrm{U}_{12}$ \\
\hline $\mathrm{B}(1)$ & $20(7)$ & $35(5)$ & $35(5)$ & $1(4)$ & $-11(5)$ & $-24(5)$ \\
\hline $\mathrm{O}(1)$ & $52(4)$ & $54(3)$ & $37(3)$ & $-21(3)$ & $0(3)$ & $8(3)$ \\
\hline $\mathrm{O}(2)$ & $39(4)$ & $54(3)$ & $50(3)$ & $-23(3)$ & $-13(3)$ & $11(3)$ \\
\hline $\mathrm{O}(3)$ & $25(4)$ & $37(3)$ & $32(3)$ & $-13(2)$ & $-6(2)$ & $4(3)$ \\
\hline $\mathrm{O}(4)$ & $23(4)$ & $52(3)$ & $46(3)$ & $-16(3)$ & $-12(2)$ & $9(3)$ \\
\hline $\mathrm{O}(5)$ & $29(4)$ & $65(4)$ & $50(3)$ & $-29(3)$ & $-18(3)$ & $4(3)$ \\
\hline$C(1)$ & $69(7)$ & $59(5)$ & $53(5)$ & $-28(4)$ & $9(4)$ & $15(5)$ \\
\hline $\mathrm{C}(2)$ & $32(6)$ & $33(4)$ & $35(4)$ & $-4(4)$ & $-2(4)$ & $-15(4)$ \\
\hline$C(3)$ & $23(5)$ & $35(4)$ & $29(4)$ & $-4(3)$ & $0(4)$ & $-10(4)$ \\
\hline$C(4)$ & $39(6)$ & $40(5)$ & $22(4)$ & $-7(3)$ & $4(4)$ & $-6(4)$ \\
\hline$C(5)$ & $27(5)$ & $40(4)$ & $22(4)$ & $-8(3)$ & $-1(3)$ & $-12(4)$ \\
\hline$C(6)$ & $23(6)$ & $59(5)$ & $35(4)$ & $-6(4)$ & $-18(4)$ & $-12(5)$ \\
\hline$C(7)$ & $97(9)$ & $83(7)$ & $73(6)$ & $27(5)$ & $-55(6)$ & $-8(6)$ \\
\hline$C(8)$ & $50(7)$ & $167(10)$ & $93(7)$ & $-78(7)$ & $-37(6)$ & $17(7)$ \\
\hline $\mathrm{C}(9)$ & $30(6)$ & $113(7)$ & $47(5)$ & $2(5)$ & $-16(4)$ & $-12(6)$ \\
\hline$C(10)$ & $23(5)$ & $44(5)$ & $30(4)$ & $-3(4)$ & $-7(4)$ & $9(4)$ \\
\hline $\mathrm{C}(11)$ & $15(5)$ & $27(4)$ & $38(4)$ & $-6(3)$ & $-10(4)$ & $-1(4)$ \\
\hline$C(12)$ & $28(6)$ & $33(4)$ & $32(4)$ & $-5(3)$ & $-13(4)$ & $-8(4)$ \\
\hline$C(13)$ & $24(5)$ & $38(5)$ & $20(4)$ & $-4(3)$ & $1(3)$ & $-12(4)$ \\
\hline$C(14)$ & $25(5)$ & $29(4)$ & $22(4)$ & $-2(3)$ & $4(3)$ & 2(4) \\
\hline$C(15)$ & $20(5)$ & $31(4)$ & $37(4)$ & $-8(3)$ & $-14(3)$ & $15(4)$ \\
\hline$C(16)$ & $25(6)$ & $83(6)$ & $33(4)$ & $-19(4)$ & $5(4)$ & $11(5)$ \\
\hline$C(17)$ & $45(6)$ & $41(5)$ & $42(4)$ & $-8(4)$ & $-13(4)$ & $10(4)$ \\
\hline C(18) & $36(6)$ & $39(5)$ & $37(4)$ & $-17(4)$ & $6(4)$ & $0(4)$ \\
\hline C(19) & $32(6)$ & $34(4)$ & $23(4)$ & $3(3)$ & $-9(3)$ & $-6(4)$ \\
\hline$C(20)$ & $39(6)$ & $55(5)$ & $30(4)$ & $-15(4)$ & $-9(4)$ & $-16(5)$ \\
\hline$C(21)$ & $106(9)$ & 142(9) & $54(6)$ & $-49(6)$ & $20(6)$ & $-59(8)$ \\
\hline$C(22)$ & $76(8)$ & $142(9)$ & $79(7)$ & $-68(6)$ & $-42(6)$ & $19(7)$ \\
\hline$C(23)$ & 113(9) & $65(6)$ & $67(6)$ & $-39(5)$ & $-30(5)$ & $-2(6)$ \\
\hline$C(24)$ & $22(5)$ & $30(4)$ & $40(4)$ & $0(3)$ & $-7(4)$ & $-3(4)$ \\
\hline$C(25)$ & $17(5)$ & $29(4)$ & $45(5)$ & $-15(4)$ & $-5(4)$ & $-13(4)$ \\
\hline
\end{tabular}


Table S2.6. Hydrogen coordinates $\left(\times 10^{4}\right)$ and isotropic displacement parameters $\left(\AA^{2} \times\right.$ $10^{3}$ ) for 5-(boronic acid)-2,7-di-tert-butyl-9,9-dimethyl-9H-xanthene-4-carboxylic acid methyl ester (24).

\begin{tabular}{|c|c|c|c|c|}
\hline Atom & $\mathrm{x}$ & $\mathrm{y}$ & $\mathrm{z}$ & $\mathrm{U}(\mathrm{eq})$ \\
\hline $\mathrm{H}(4)$ & 12395 & 5433 & 4611 & 61 \\
\hline $\mathrm{H}(5)$ & 14177 & 6123 & 6753 & 68 \\
\hline $\mathrm{H}(1 \mathrm{~A})$ & 12752 & 5058 & 1375 & 94 \\
\hline $\mathrm{H}(1 \mathrm{~B})$ & 11780 & 4767 & 544 & 94 \\
\hline $\mathrm{H}(1 \mathrm{C})$ & 11867 & 3935 & 1733 & 94 \\
\hline $\mathrm{H}(4 \mathrm{~A})$ & 8039 & 6456 & 1821 & 41 \\
\hline $\mathrm{H}(7 \mathrm{~A})$ & 6050 & 9481 & 1065 & 132 \\
\hline $\mathrm{H}(7 \mathrm{~B})$ & 5025 & 9753 & 2110 & 132 \\
\hline $\mathrm{H}(7 \mathrm{C})$ & 4321 & 9541 & 1099 & 132 \\
\hline $\mathrm{H}(8 \mathrm{~A})$ & 4699 & 7461 & 665 & 145 \\
\hline $\mathrm{H}(8 \mathrm{~B})$ & 5585 & 6310 & 1422 & 145 \\
\hline $\mathrm{H}(8 \mathrm{C})$ & 6435 & 7323 & 653 & 145 \\
\hline $\mathrm{H}(9 \mathrm{~A})$ & 3074 & 7948 & 2300 & 98 \\
\hline $\mathrm{H}(9 \mathrm{~B})$ & 3794 & 8071 & 3338 & 98 \\
\hline $\mathrm{H}(9 \mathrm{C})$ & 3973 & 6768 & 3016 & 98 \\
\hline $\mathrm{H}(10)$ & 5798 & 8530 & 3862 & 41 \\
\hline $\mathrm{H}(16 \mathrm{~A})$ & 5474 & 8300 & 5754 & 74 \\
\hline $\mathrm{H}(16 \mathrm{~B})$ & 6304 & 8393 & 6751 & 74 \\
\hline $\mathrm{H}(16 \mathrm{C})$ & 6507 & 7125 & 6360 & 74 \\
\hline $\mathrm{H}(17 \mathrm{~A})$ & 6648 & 10112 & 4519 & 66 \\
\hline $\mathrm{H}(17 \mathrm{~B})$ & 8384 & 10031 & 4438 & 66 \\
\hline $\mathrm{H}(17 \mathrm{C})$ & 7430 & 10222 & 5533 & 66 \\
\hline $\mathrm{H}(18)$ & 8503 & 8936 & 7116 & 45 \\
\hline $\mathrm{H}(21 \mathrm{~A})$ & 9481 & 7234 & 9564 & 140 \\
\hline $\mathrm{H}(21 \mathrm{~B})$ & 8380 & 8409 & 9035 & 140 \\
\hline $\mathrm{H}(21 \mathrm{C})$ & 9312 & 8480 & 9985 & 140 \\
\hline $\mathrm{H}(22 \mathrm{~A})$ & 12039 & 8470 & 9646 & 140 \\
\hline $\mathrm{H}(22 \mathrm{~B})$ & 12848 & 8354 & 8486 & 140 \\
\hline $\mathrm{H}(22 \mathrm{C})$ & 12215 & 7210 & 9247 & 140 \\
\hline $\mathrm{H}(23 \mathrm{~A})$ & 9401 & 10281 & 7869 & 116 \\
\hline
\end{tabular}


X-tal Structural data for $\mathbf{2 4}$

\begin{tabular}{lrrrr}
$\mathrm{H}(23 \mathrm{~B})$ & 11132 & 10246 & 7687 & 116 \\
$\mathrm{H}(23 \mathrm{C})$ & 10303 & 10302 & 8852 & 116 \\
$\mathrm{H}(24)$ & 12554 & 6989 & 7420 & 38 \\
\hline
\end{tabular}


Table S3.1. Bond distance parameters from DFT calculated energy minimized structures of the hydroperoxide complexes of $\mathrm{Mn}\left(\mathrm{HSX}^{*}\right)$ and $\mathrm{Mn}\left(\mathrm{H}_{\mathrm{ph}} \mathrm{SX}^{*}\right)$.

\begin{tabular}{lcc}
\hline Distance $(\AA)$ & $\mathrm{Mn}\left(\mathrm{HSX}^{*}\right) \mathrm{OOH}$ & $\mathrm{Mn}\left(\mathrm{H}_{\mathrm{ph}} \mathrm{SX}\right) * \mathrm{OOH}$ \\
\hline Mn-O & $1.89 \AA$ & $1.85 \AA$ \\
peroxide O-O & $1.46 \AA$ & $1.46 \AA$ \\
peroxide O-H & 0.99 & $0.99 \AA$ \\
acid OH- Mn & $5.14 \AA, 6.77 \AA$ & $4.58 \AA, 6.03 \AA$ \\
& peroxide $\mathbf{H}$ to acid $\mathbf{O H}:$ & peroxide $\mathbf{H}$ to acid CO: \\
peroxide-acid & $1.94 \AA$ & $3.11 \AA$ \\
acid dimer (OH-CO) & no dimer & $2.57 \AA, 2.68 \AA$ \\
\hline
\end{tabular}


Table S3.2 Total bond energies of calculated compounds.

\begin{tabular}{lc}
\hline & Total Energy $(\mathrm{kJ} / \mathrm{mol})$ \\
\hline $\mathrm{Mn}\left(\mathrm{HSX}^{*}\right)-\mathrm{OOH}$ & -56695.92 \\
$\mathrm{Mn}\left(\mathrm{HphSX}^{*}\right)-\mathrm{OOH}$ (carboxylic acid dimer) & -69749.31 \\
$\mathrm{Mn}\left(\mathrm{HphSX}^{*}\right)-\mathrm{OOH}$ (no carboxylic acid dimer) & -69704.88 \\
\hline
\end{tabular}


Table S3.3 Cartesian coordinates from the DFT calculated energy minimized structure of the hydroperoxide complex of $\mathrm{Mn}\left(\mathrm{HSX}^{*}\right)$. The structure is shown in Figure 3(a).

\begin{tabular}{|c|c|c|c|}
\hline & $\mathrm{x}$ & $\mathrm{y}$ & $\mathrm{z}$ \\
\hline 1.C & 10.54703 & -3.38522 & -3.3624 \\
\hline 2.C & 9.68632 & -2.64592 & -2.55441 \\
\hline 3.C & 8.311687 & -2.76836 & -2.75099 \\
\hline 4.C & 7.783106 & -3.63145 & -3.72707 \\
\hline 5.C & 8.682754 & -4.36068 & -4.51001 \\
\hline 6.C & 10.05356 & -4.24241 & -4.33893 \\
\hline 7.0 & 7.416122 & -2.0485 & -1.96717 \\
\hline 8.C & 7.862076 & -0.94236 & -1.24597 \\
\hline 9.C & 9.218721 & -0.75202 & -0.9971 \\
\hline 10.C & 10.21893 & -1.77446 & -1.45312 \\
\hline 11.C & 6.884345 & -0.0688 & -0.7487 \\
\hline 12.C & 7.324951 & 1.027343 & 0.001137 \\
\hline 13.C & 8.675675 & 1.254335 & 0.240083 \\
\hline 14.C & 9.615952 & 0.364363 & -0.26163 \\
\hline 15.C & 5.427121 & -0.25561 & -0.94458 \\
\hline 16.C & 4.814239 & -0.16331 & -2.2162 \\
\hline 17.C & 3.44543 & -0.20198 & -2.36716 \\
\hline 18.C & 2.57427 & -0.34933 & -1.24733 \\
\hline 19.C & 3.203745 & -0.47449 & 0.049393 \\
\hline 20.C & 4.608608 & -0.41023 & 0.159554 \\
\hline 21.0 & 1.287077 & -0.34475 & -1.42562 \\
\hline 22.Mn & -0.16069 & -0.86555 & -0.23384 \\
\hline 23.0 & -0.6646 & -2.5896 & 0.3577 \\
\hline 24.0 & -2.07419 & -2.96625 & 0.295007 \\
\hline 25.C & 2.429979 & -0.60886 & 1.24072 \\
\hline 26.N & 1.137132 & -0.75186 & 1.294269 \\
\hline 27.C & 0.434907 & -0.76124 & 2.575628 \\
\hline 28.C & -0.67066 & 0.304545 & 2.506114 \\
\hline 29.N & -1.35727 & 0.138633 & 1.233124 \\
\hline $30 . \mathrm{C}$ & -2.63639 & 0.283297 & 1.136077 \\
\hline 31.C & -3.39444 & 0.07964 & -0.06945 \\
\hline
\end{tabular}




\begin{tabular}{|c|c|c|c|}
\hline 32.C & -2.80265 & -0.30302 & -1.316 \\
\hline 33.C & -3.66507 & -0.45679 & -2.42489 \\
\hline 34.C & -5.03447 & -0.30466 & -2.30187 \\
\hline $35 . \mathrm{C}$ & -5.62856 & 0.015866 & -1.0648 \\
\hline 36.C & -4.79101 & 0.222814 & 0.020031 \\
\hline 37.0 & -1.51506 & -0.5233 & -1.49761 \\
\hline $38 . \mathrm{C}$ & -7.09904 & 0.10156 & -0.94635 \\
\hline 39.C & -7.83231 & 0.931162 & -1.80171 \\
\hline 40.C & -9.22335 & 0.979313 & -1.7475 \\
\hline 41.C & -9.90188 & 0.188831 & -0.82458 \\
\hline 42.C & -9.20428 & -0.64331 & 0.053971 \\
\hline 43.C & -7.81255 & -0.67904 & -0.01552 \\
\hline 44.0 & -7.0592 & -1.48972 & 0.813131 \\
\hline 45.C & -7.65478 & -2.53137 & 1.48274 \\
\hline 46.C & -9.04792 & -2.58636 & 1.623098 \\
\hline 47.C & -9.91558 & -1.47852 & 1.081779 \\
\hline 48.C & -6.83248 & -3.54502 & 2.033475 \\
\hline 49.C & -7.45473 & -4.59552 & 2.719975 \\
\hline $50 . \mathrm{C}$ & -8.8336 & -4.66286 & 2.858963 \\
\hline 51.C & -9.62136 & -3.65903 & 2.303248 \\
\hline 52.C & -5.33755 & -3.66437 & 1.922399 \\
\hline 53.0 & -4.73639 & -2.61443 & 1.329674 \\
\hline 54.C & 6.320222 & -3.86776 & -4.03141 \\
\hline 55.0 & 5.401275 & -3.2661 & -3.24738 \\
\hline 56.0 & -4.73514 & -4.64188 & 2.338189 \\
\hline 57.0 & 5.975872 & -4.58544 & -4.94947 \\
\hline 58.H & -3.77078 & -2.82908 & 1.23245 \\
\hline 59.H & 5.849483 & -2.71298 & -2.56649 \\
\hline $60 . \mathrm{H}$ & 8.254883 & -5.0207 & -5.26105 \\
\hline 61.H & 10.73623 & -4.81956 & -4.95944 \\
\hline $62 . \mathrm{H}$ & 11.62155 & -3.29034 & -3.20684 \\
\hline 63.H & 10.49567 & -2.41122 & -0.59286 \\
\hline 64.H & 11.13619 & -1.27075 & -1.78935 \\
\hline $65 . \mathrm{H}$ & 10.67786 & 0.520843 & -0.07428 \\
\hline $66 . \mathrm{H}$ & 8.991218 & 2.126241 & 0.809769 \\
\hline 67.H & 6.575785 & 1.725685 & 0.37136 \\
\hline
\end{tabular}




\begin{tabular}{lccc}
$68 . \mathrm{H}$ & -6.8036 & -5.36913 & 3.125449 \\
$69 . \mathrm{H}$ & -9.29376 & -5.49536 & 3.391576 \\
$70 . \mathrm{H}$ & -10.7081 & -3.69612 & 2.401853 \\
$71 . \mathrm{H}$ & -10.992 & 0.218998 & -0.77225 \\
$72 . \mathrm{H}$ & -9.77516 & 1.637244 & -2.41979 \\
$73 . \mathrm{H}$ & -7.28691 & 1.557318 & -2.5098 \\
$74 . \mathrm{H}$ & -10.8272 & -1.91062 & 0.638539 \\
$75 . \mathrm{H}$ & -10.2388 & -0.82912 & 1.918061 \\
$76 . \mathrm{H}$ & -3.2148 & -0.72904 & -3.38048 \\
$77 . \mathrm{H}$ & -5.6749 & -0.47482 & -3.1698 \\
$78 . \mathrm{H}$ & -5.22411 & 0.485137 & 0.985485 \\
$79 . \mathrm{H}$ & 2.988855 & -0.11889 & -3.3517 \\
$80 . \mathrm{H}$ & 5.442582 & -0.03236 & -3.09736 \\
$81 . \mathrm{H}$ & 5.059577 & -0.49095 & 1.149005 \\
$82 . \mathrm{H}$ & 1.121107 & -0.55949 & 3.414924 \\
$83 . \mathrm{H}$ & -0.02263 & -1.75599 & 2.701444 \\
$84 . \mathrm{H}$ & -0.20891 & 1.308071 & 2.529538 \\
$85 . \mathrm{H}$ & -1.35302 & 0.208945 & 3.369173 \\
$86 . \mathrm{H}$ & -2.1853 & -3.16188 & -0.66379 \\
$87 . \mathrm{H}$ & 2.982576 & -0.57186 & 2.192149 \\
$88 . \mathrm{H}$ & -3.22249 & 0.563181 & 2.027737 \\
\hline & & & \\
\hline & & & \\
\hline & & & \\
\hline
\end{tabular}


Table S3.4 Cartesian coordinates from the DFT calculated energy minimized structure of the hydroperoxide complex of $\mathrm{Mn}\left(\mathrm{H}_{\mathrm{ph}} \mathrm{SX}^{*}\right)$ with the xanthenes oriented so the carboxylic acids can form a hydrogen bonded dimer. The structure is shown in Figure 3(b).

\begin{tabular}{llll}
\hline & $\mathrm{x}$ & $\mathrm{y}$ & $\mathrm{z}$ \\
\hline $1 . \mathrm{C}$ & -3.75165 & 4.546489 & 14.31793 \\
2.C & -3.38406 & 3.626824 & 15.30982 \\
3.C & -2.8434 & 2.395334 & 14.92456 \\
4.C & -2.68628 & 2.083854 & 13.58282 \\
5.C & -3.06025 & 3.004904 & 12.59827 \\
6.C & -3.58758 & 4.242615 & 12.97275 \\
7.C & -3.60206 & 3.958867 & 16.73365 \\
8.C & -2.56721 & 3.891009 & 17.68142 \\
9.C & -2.77102 & 4.23596 & 19.01614 \\
10.C & -4.04147 & 4.653395 & 19.41208 \\
11.C & -5.08914 & 4.71473 & 18.49963 \\
12.C & -4.86443 & 4.370164 & 17.17086 \\
13.C & -1.63375 & 4.113736 & 19.99311 \\
14.C & -0.29351 & 4.125105 & 19.30752 \\
$15 . C$ & -0.20586 & 3.78953 & 17.95878 \\
16.O & -1.33477 & 3.477823 & 17.22283 \\
17.C & 1.021914 & 3.710811 & 17.27923 \\
18.C & 2.176114 & 3.978908 & 18.02141 \\
19.C & 2.115825 & 4.326536 & 19.3671 \\
20.C & 0.882194 & 4.40687 & 20.00279 \\
21.C & 1.11115 & 3.36242 & 15.84516 \\
22.C & 0.330315 & 4.025557 & 14.87198 \\
23.C & 3.706708 & 13.53512 \\
24.C & 2.691984 & 13.06558 \\
25.C & 2.065424 & 14.03725 \\
26.C & 2.405781 & 15.40133 \\
27.C & 1.281166 & 0.125374 & 13.65775 \\
28.N & 2.13297 & -0.26124 & 12.44032 \\
29.C & 2.009736 & & 12.15586 \\
30.C & 3.137512 & 11.08788 \\
& 3.331359 &
\end{tabular}




\begin{tabular}{|c|c|c|c|}
\hline 31.N & 3.242431 & -0.423 & 10.04361 \\
\hline $32 . \mathrm{C}$ & 3.509082 & -0.6284 & 8.784802 \\
\hline $33 . \mathrm{C}$ & 3.007294 & 0.125546 & 7.684865 \\
\hline $34 . C$ & 2.209732 & 1.311089 & 7.875835 \\
\hline $35 . \mathrm{C}$ & 1.736861 & 1.958986 & 6.698018 \\
\hline $36 . C$ & 2.071322 & 1.513461 & 5.438487 \\
\hline 37.C & 2.928479 & 0.399599 & 5.246521 \\
\hline $38 . C$ & 3.364036 & -0.27635 & 6.374625 \\
\hline 39.C & 3.459637 & 0.040274 & 3.909948 \\
\hline 40.C & 2.648788 & -0.24499 & 2.794942 \\
\hline 41.C & 3.186567 & -0.58831 & 1.553547 \\
\hline 42.C & 4.57522 & -0.60557 & 1.405997 \\
\hline 43.C & 5.406733 & -0.31751 & 2.483065 \\
\hline 44.C & 4.846457 & -0.00795 & 3.720003 \\
\hline 45.C & 2.279383 & -0.99611 & 0.421165 \\
\hline 46.C & 0.856353 & -0.55775 & 0.641278 \\
\hline 47.C & 0.431035 & -0.20078 & 1.921345 \\
\hline 48.0 & 1.284142 & -0.19645 & 3.002532 \\
\hline 49.C & -0.89868 & 0.185909 & 2.177049 \\
\hline $50 . \mathrm{C}$ & -1.79941 & 0.201045 & 1.107698 \\
\hline 51.C & -1.39444 & -0.15816 & -0.17648 \\
\hline $52 . \mathrm{C}$ & -0.07203 & -0.5304 & -0.40197 \\
\hline 53.C & -1.31587 & 0.571328 & 3.542132 \\
\hline 54.C & -1.92146 & 1.812961 & 3.783046 \\
\hline 55.C & -2.25587 & 2.200459 & 5.077877 \\
\hline 56.C & -1.98531 & 1.343799 & 6.154086 \\
\hline 57.C & -1.41963 & 0.086463 & 5.90944 \\
\hline 58.C & -1.09142 & -0.29527 & 4.619417 \\
\hline 59.0 & 1.932871 & 1.80829 & 9.040583 \\
\hline 60.Mn & 2.017818 & 0.941913 & 10.83467 \\
\hline 61.0 & 1.273912 & 2.407007 & 11.78954 \\
\hline 62.C & -2.24824 & 1.724205 & 7.56468 \\
\hline 63.0 & -2.774 & 2.917394 & 7.745202 \\
\hline 64.C & -2.88906 & 2.675209 & 11.16074 \\
\hline 65.0 & -2.53684 & 1.425547 & 10.95134 \\
\hline 66.0 & 0.535003 & -0.20666 & 11.02656 \\
\hline
\end{tabular}




\begin{tabular}{|c|c|c|c|}
\hline 67.0 & 0.250044 & -0.84851 & 9.744538 \\
\hline 68.0 & -1.96951 & 0.937607 & 8.494387 \\
\hline 69.0 & -3.06877 & 3.51929 & 10.25959 \\
\hline $70 . \mathrm{H}$ & -2.83754 & 0.474442 & 1.302656 \\
\hline 71.H & -2.11176 & -0.15753 & -0.99779 \\
\hline $72 . \mathrm{H}$ & 0.252183 & -0.81884 & -1.40393 \\
\hline $73 . \mathrm{H}$ & 2.306334 & -2.09848 & 0.319297 \\
\hline 74.H & 2.655165 & -0.57658 & -0.52584 \\
\hline $75 . \mathrm{H}$ & 5.000639 & -0.85511 & 0.432238 \\
\hline 76.H & 6.4899 & -0.31843 & 2.358736 \\
\hline 76.H & 6.4899 & -0.31843 & 2.358736 \\
\hline 77.H & 5.487723 & 0.252168 & 4.563406 \\
\hline 78.H & -5.67994 & 4.39356 & 16.44961 \\
\hline 79.H & -6.08157 & 5.022087 & 18.8235 \\
\hline $80 . \mathrm{H}$ & -4.20853 & 4.91942 & 20.45552 \\
\hline 81.H & 0.822128 & 4.681388 & 21.05555 \\
\hline 82.H & 3.03054 & 4.549584 & 19.91288 \\
\hline 83.H & 3.136077 & 3.953563 & 17.50816 \\
\hline 84.H & -1.68326 & 4.932077 & 20.72549 \\
\hline $85 . \mathrm{H}$ & -1.74167 & 3.168577 & 20.55642 \\
\hline 86.H & -0.19761 & 4.225949 & 12.79949 \\
\hline 87.H & -0.35209 & 4.813256 & 15.18598 \\
\hline 88.H & 2.633856 & 1.880058 & 16.12387 \\
\hline 89.H & 1.111855 & 2.843248 & 6.830108 \\
\hline $90 . \mathrm{H}$ & 1.697964 & 2.051326 & 4.565478 \\
\hline 91.H & 3.999387 & -1.15685 & 6.253866 \\
\hline $92 . \mathrm{H}$ & 4.684173 & -1.8534 & 10.68829 \\
\hline 93.H & 3.095807 & -1.88001 & 11.53033 \\
\hline 94.H & 5.264114 & 0.286524 & 11.75112 \\
\hline 95.H & 4.711448 & -0.81151 & 13.059 \\
\hline 96.H & -0.41679 & -0.22796 & 9.366585 \\
\hline 97.H & -2.88509 & 3.120288 & 8.762245 \\
\hline 98.H & -0.6426 & -1.2716 & 4.439241 \\
\hline 99.H & -1.23114 & -0.58331 & 6.745625 \\
\hline 100.H & -2.0949 & 2.494781 & 2.94807 \\
\hline 101.H & -2.70507 & 3.176113 & 5.260958 \\
\hline
\end{tabular}




\begin{tabular}{lccc}
$102 . \mathrm{H}$ & -2.34413 & 1.271822 & 9.951154 \\
$103 . \mathrm{H}$ & -2.26015 & 1.128916 & 13.28726 \\
$104 . \mathrm{H}$ & -2.54897 & 1.674658 & 15.68419 \\
$105 . \mathrm{H}$ & -4.15206 & 5.516006 & 14.61272 \\
$106 . \mathrm{H}$ & -3.86272 & 4.958224 & 12.20067 \\
$107 . \mathrm{H}$ & 4.190518 & -1.45728 & 8.5362 \\
$108 . \mathrm{H}$ & 3.779046 & 0.736605 & 14.46404 \\
\hline
\end{tabular}




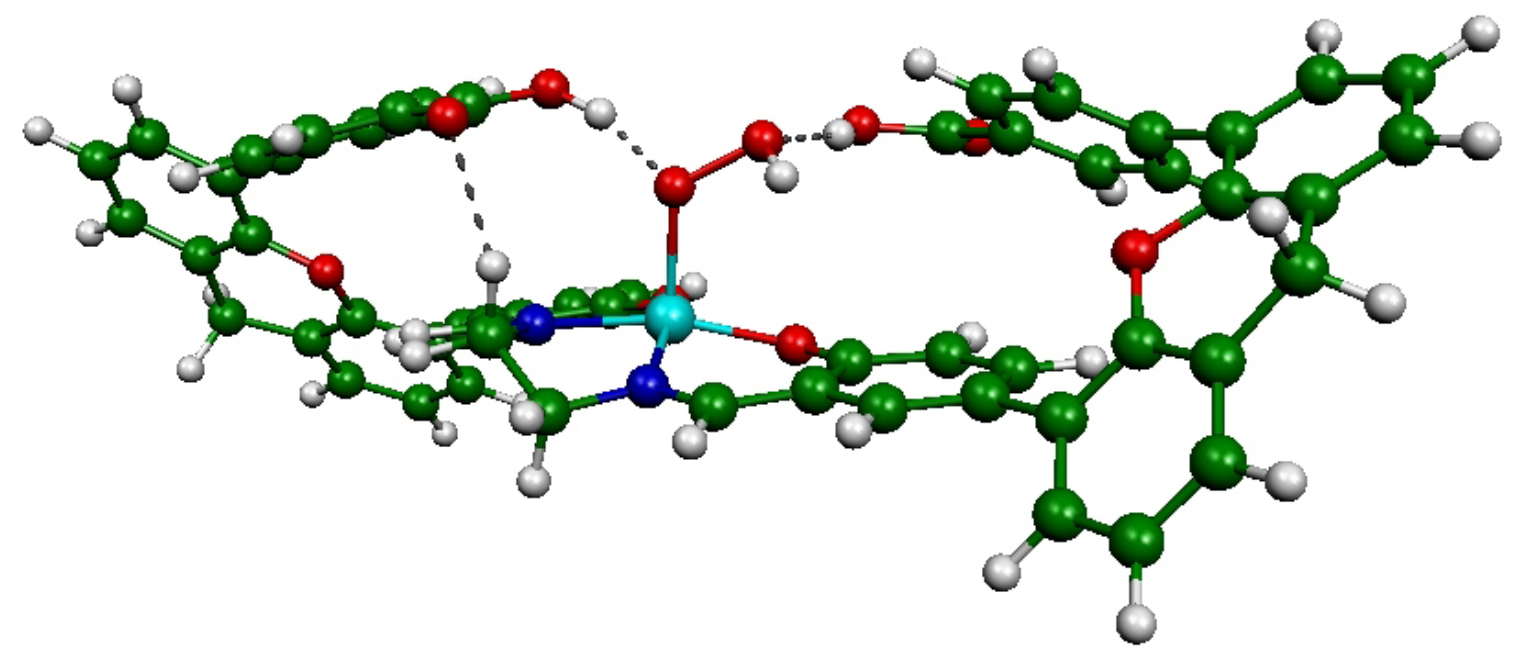

Figure S3.1 Energy minimized structure obtained from DFT of the hydroperoxide complexes of $\mathrm{Mn}\left(\mathrm{H}_{\mathrm{ph}} \mathrm{SX}^{*}-\mathrm{COOH}\right)$ with the xanthenes splayed away from each other, precluding formation of the $-(\mathrm{COOH})_{2}-$ dimer. Each of the carboxylic acids has a hydrogen- bonding interaction with the hydroperoxide. 
Table S3.5 Cartesian coordinates from the DFT calculated energy minimized structure of the hydroperoxide complex of $\mathrm{Mn}\left(\mathrm{H}_{\mathrm{ph}} \mathrm{SX}^{*}\right)$ with the xanthenes splayed away from each other, precluding formation of the $-(\mathrm{COOH})_{2}-$ dimer. (The structure is shown in Figure S3.1)

\begin{tabular}{|c|c|c|c|}
\hline & $\mathrm{X}$ & $\mathrm{y}$ & $\mathrm{Z}$ \\
\hline 1.C & 0.318602 & 5.619625 & 15.67877 \\
\hline 2.C & -1.03035 & 5.288744 & 15.85218 \\
\hline 3.C & -1.80647 & 5.010826 & 14.72388 \\
\hline 4.C & -1.24529 & 5.050496 & 13.45124 \\
\hline 5.C & 0.107431 & 5.358143 & 13.2815 \\
\hline 6.C & 0.878647 & 5.657568 & 14.40875 \\
\hline 7.C & -1.6201 & 5.278902 & 17.21186 \\
\hline 8.C & -2.11217 & 4.100881 & 17.78755 \\
\hline 9.C & -2.64949 & 4.071659 & 19.07316 \\
\hline 10.C & -2.68154 & 5.255792 & 19.80812 \\
\hline 11.C & -2.19993 & 6.44201 & 19.26403 \\
\hline 12.C & -1.67672 & 6.448512 & 17.974 \\
\hline 13.C & -3.20089 & 2.774928 & 19.59977 \\
\hline 14.C & -2.52797 & 1.59271 & 18.95657 \\
\hline 15.C & -1.98332 & 1.738529 & 17.68226 \\
\hline 16.0 & -2.02149 & 2.951232 & 17.02309 \\
\hline 17.C & -1.41374 & 0.660886 & 16.98784 \\
\hline 18.C & -1.40223 & -0.58576 & 17.62143 \\
\hline 19.C & -1.92735 & -0.75141 & 18.89896 \\
\hline 20.C & -2.48304 & 0.33765 & 19.56226 \\
\hline 21.C & -0.87982 & 0.807457 & 15.61754 \\
\hline 22.C & 0.104325 & 1.763175 & 15.2795 \\
\hline 23.C & 0.607953 & 1.866599 & 13.99913 \\
\hline 24.C & 0.134787 & 1.043494 & 12.95146 \\
\hline 25.C & -0.84444 & 0.056638 & 13.29364 \\
\hline 26.C & -1.3209 & -0.03865 & 14.61848 \\
\hline 27.C & -1.31766 & -0.88833 & 12.34079 \\
\hline 28.N & -1.0586 & -0.86453 & 11.0687 \\
\hline 29.C & -1.54578 & -1.93995 & 10.20082 \\
\hline
\end{tabular}




\begin{tabular}{|c|c|c|c|}
\hline $30 . \mathrm{C}$ & -1.84123 & -1.30742 & 8.848462 \\
\hline $31 . \mathrm{N}$ & -0.68379 & -0.46505 & 8.531889 \\
\hline 32.C & -0.11339 & -0.54823 & 7.362761 \\
\hline $33 . \mathrm{C}$ & 1.03234 & 0.181635 & 6.943407 \\
\hline 34.C & 1.653 & 1.157236 & 7.788614 \\
\hline $35 . C$ & 2.751387 & 1.868368 & 7.240496 \\
\hline 36.C & 3.238248 & 1.570288 & 5.983477 \\
\hline $37 . C$ & 2.684113 & 0.54916 & 5.169879 \\
\hline $38 . C$ & 1.567746 & -0.1046 & 5.666886 \\
\hline 39.C & 3.344486 & 0.149931 & 3.905987 \\
\hline 40.C & 2.643765 & -0.23746 & 2.741226 \\
\hline 41.C & 3.303061 & -0.66561 & 1.587981 \\
\hline 42.C & 4.699641 & -0.68156 & 1.578923 \\
\hline 43.C & 5.422227 & -0.29293 & 2.701968 \\
\hline 44.C & 4.7454 & 0.106703 & 3.850936 \\
\hline 45.C & 2.520279 & -1.14983 & 0.393329 \\
\hline 46.C & 1.056677 & -0.79951 & 0.466635 \\
\hline 47.C & 0.508408 & -0.3362 & 1.661119 \\
\hline 48.0 & 1.267409 & -0.1736 & 2.80125 \\
\hline 49.C & -0.86574 & -0.05478 & 1.786744 \\
\hline $50 . C$ & -1.68432 & -0.25771 & 0.670982 \\
\hline 51.C & -1.15558 & -0.70771 & -0.53681 \\
\hline 52.C & 0.208347 & -0.97313 & -0.63097 \\
\hline 53.C & -1.43785 & 0.417976 & 3.066854 \\
\hline 54.C & -0.91968 & 1.543641 & 3.720266 \\
\hline 55.C & -1.43228 & 1.948707 & 4.94659 \\
\hline 56.C & -2.47937 & 1.236602 & 5.543434 \\
\hline 57.C & -3.03863 & 0.14502 & 4.869497 \\
\hline 58.C & -2.519 & -0.26422 & 3.646006 \\
\hline 59.0 & 1.262347 & 1.418726 & 9.005161 \\
\hline 60.Mn & -0.09393 & 0.616229 & 10.08887 \\
\hline 61.0 & 0.61384 & 1.212467 & 11.74124 \\
\hline 62.C & -2.91828 & 1.51337 & 6.949663 \\
\hline 63.0 & -2.24575 & 2.522214 & 7.507636 \\
\hline 64.C & 0.784147 & 5.441983 & 11.93933 \\
\hline 65.0 & 0.216481 & 4.806699 & 10.89687 \\
\hline
\end{tabular}




\begin{tabular}{|c|c|c|c|}
\hline 66.0 & -1.60327 & 1.950353 & 9.950737 \\
\hline 67.0 & -1.86347 & 2.95312 & 11.00138 \\
\hline 68.0 & -3.75027 & 0.810921 & 7.521208 \\
\hline 69.0 & 1.815217 & 6.075576 & 11.79406 \\
\hline $70 . \mathrm{H}$ & -2.74756 & -0.02911 & 0.75873 \\
\hline 71.H & -1.80473 & -0.85078 & -1.40125 \\
\hline $72 . \mathrm{H}$ & 0.631984 & -1.33507 & -1.57025 \\
\hline 73.H & 2.625178 & -2.24968 & 0.321945 \\
\hline 74.H & 2.954824 & -0.72685 & -0.52722 \\
\hline $75 . \mathrm{H}$ & 5.218955 & -1.01916 & 0.680064 \\
\hline 76.H & 6.512325 & -0.32059 & 2.692441 \\
\hline 77.H & 5.310193 & 0.362008 & 4.748086 \\
\hline 78.H & -1.3059 & 7.37239 & 17.53514 \\
\hline 79.H & -2.23651 & 7.361413 & 19.84392 \\
\hline $80 . \mathrm{H}$ & -3.10162 & 5.24174 & 20.81458 \\
\hline 81.H & -2.90872 & 0.213146 & 20.55823 \\
\hline 82.H & -1.90116 & -1.72979 & 19.37691 \\
\hline 83.H & -0.94288 & -1.4274 & 17.10416 \\
\hline 84.H & -3.0875 & 2.726311 & 20.69039 \\
\hline 85.H & -4.28774 & 2.729835 & 19.38454 \\
\hline 86.H & 1.378574 & 2.600403 & 13.76485 \\
\hline 87.H & 0.485305 & 2.425654 & 16.05639 \\
\hline $88 . \mathrm{H}$ & -2.08101 & -0.78558 & 14.84409 \\
\hline 89.H & 3.202437 & 2.663417 & 7.841351 \\
\hline 90.H & 4.080114 & 2.154097 & 5.592003 \\
\hline 91.H & 1.090486 & -0.88679 & 5.079501 \\
\hline 92.H & -2.00464 & -2.06467 & 8.070056 \\
\hline 93.H & -2.72927 & -0.65756 & 8.909303 \\
\hline 94.H & -0.73716 & -2.68945 & 10.09072 \\
\hline 95.H & -2.43145 & -2.42744 & 10.62344 \\
\hline 96.H & -2.09593 & 2.384333 & 11.76924 \\
\hline 97.H & -2.20382 & 2.406437 & 8.528486 \\
\hline 98.H & -2.92749 & -1.14358 & 3.143468 \\
\hline 99.H & -3.86153 & -0.39244 & 5.341844 \\
\hline 100.H & -0.09344 & 2.093599 & 3.269636 \\
\hline 101.H & -1.00029 & 2.801417 & 5.468514 \\
\hline
\end{tabular}




\begin{tabular}{lccl}
$102 . \mathrm{H}$ & -0.50246 & 4.160987 & 11.13767 \\
$103 . \mathrm{H}$ & -1.88492 & 4.879932 & 12.588 \\
$104 . \mathrm{H}$ & -2.86528 & 4.782888 & 14.84809 \\
$105 . \mathrm{H}$ & 0.930679 & 5.839595 & 16.5547 \\
$106 . \mathrm{H}$ & 1.921994 & 5.928019 & 14.26209 \\
$107 . \mathrm{H}$ & -1.93624 & -1.70839 & 12.7255 \\
$108 . \mathrm{H}$ & -0.54179 & -1.24444 & 6.631932 \\
\hline
\end{tabular}



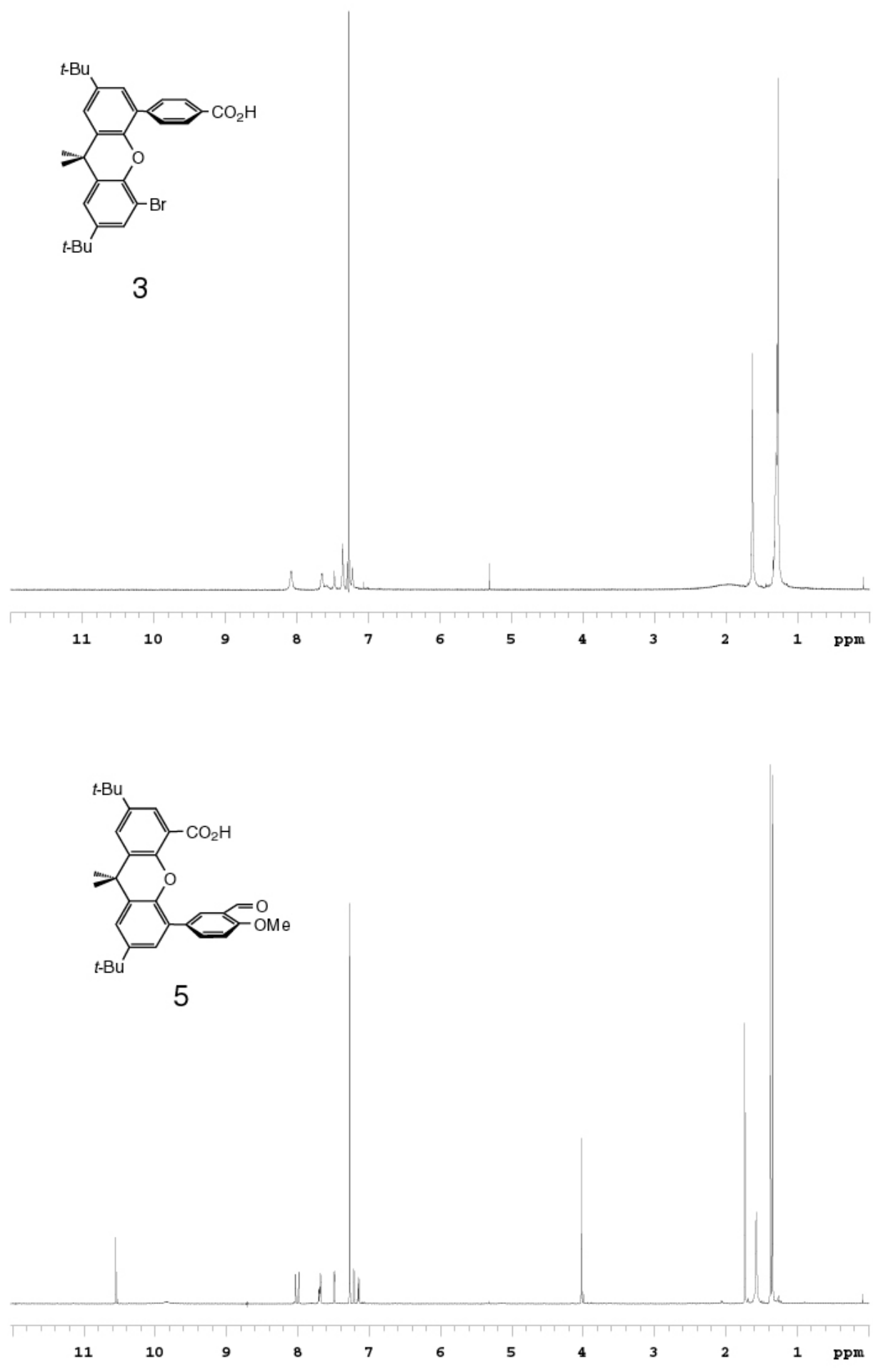


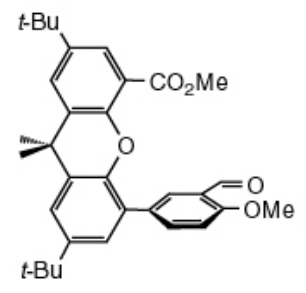

6

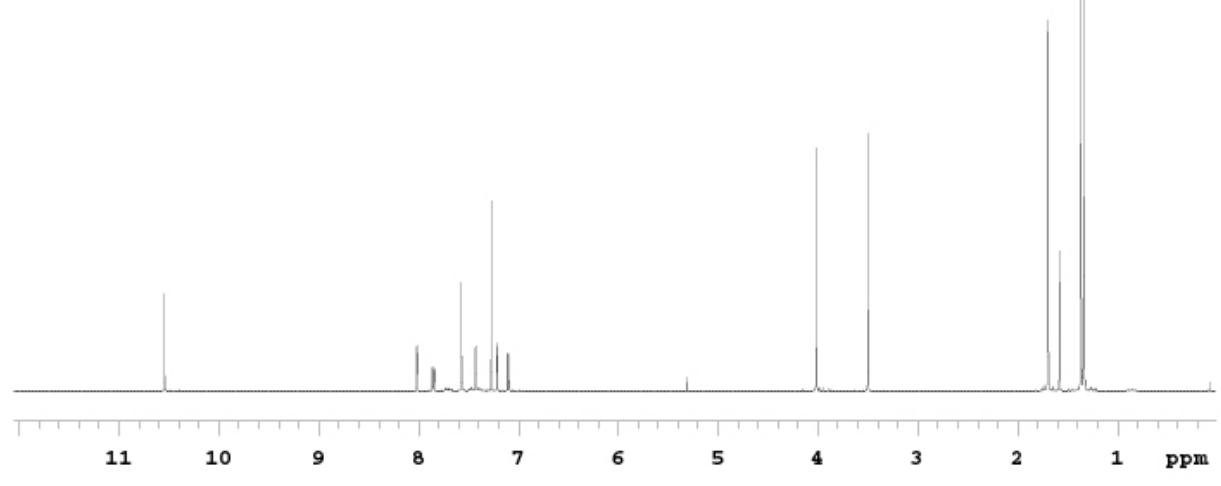




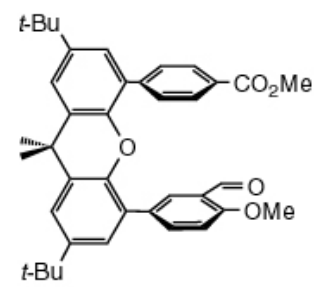

8

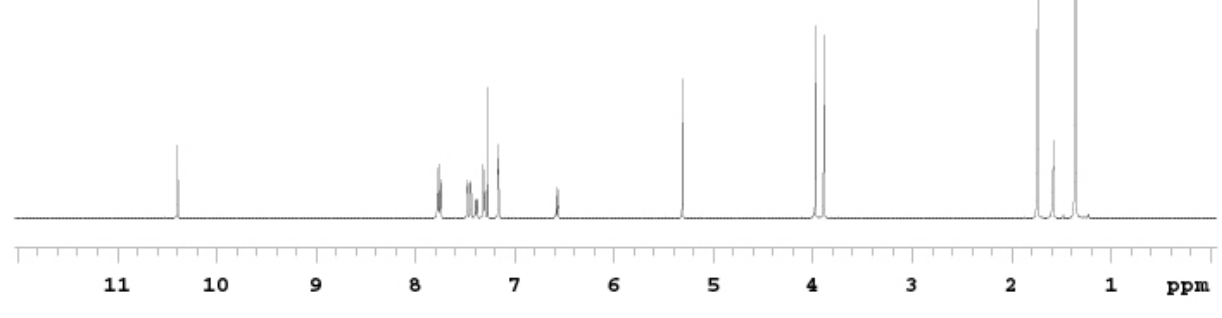




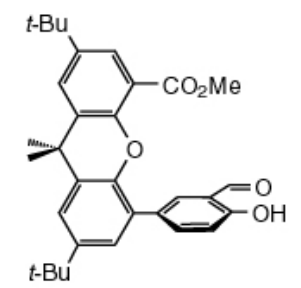

10
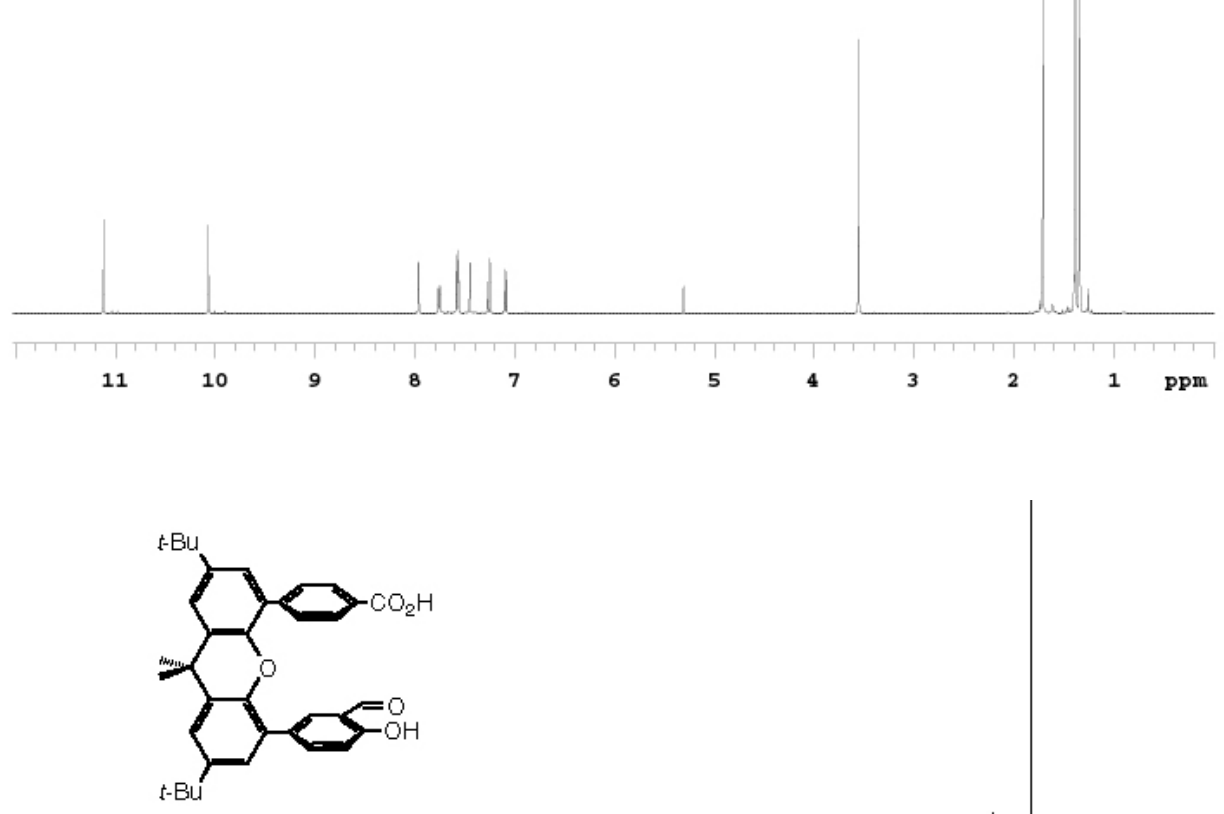

11

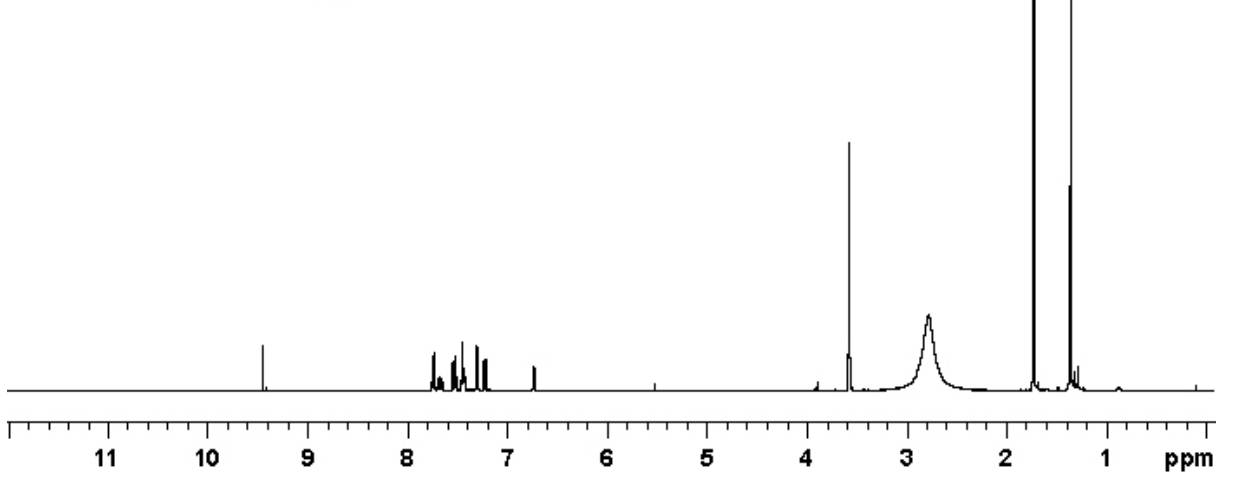




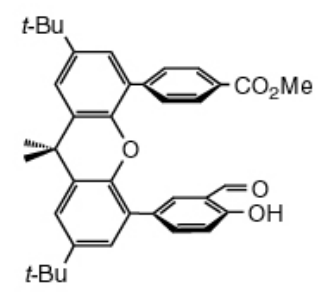

12

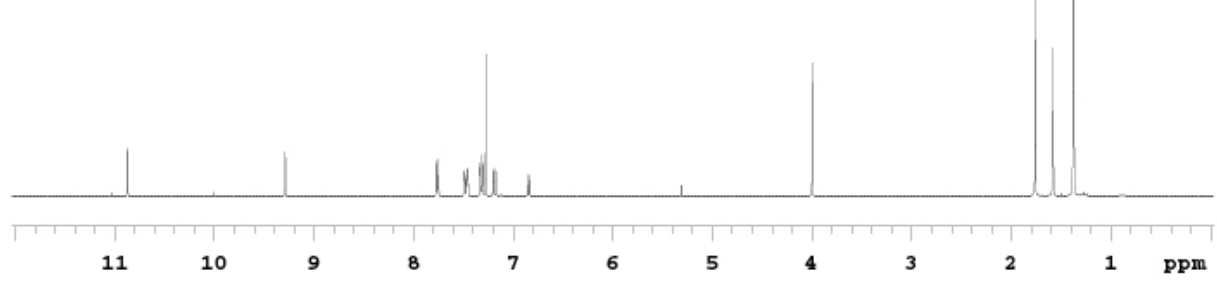




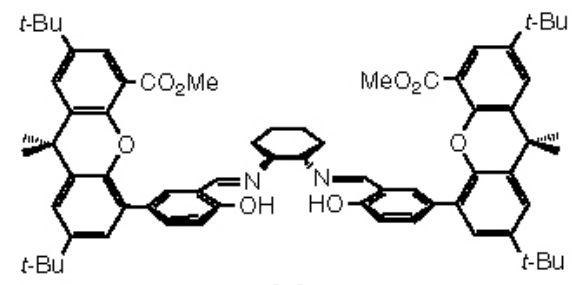

14
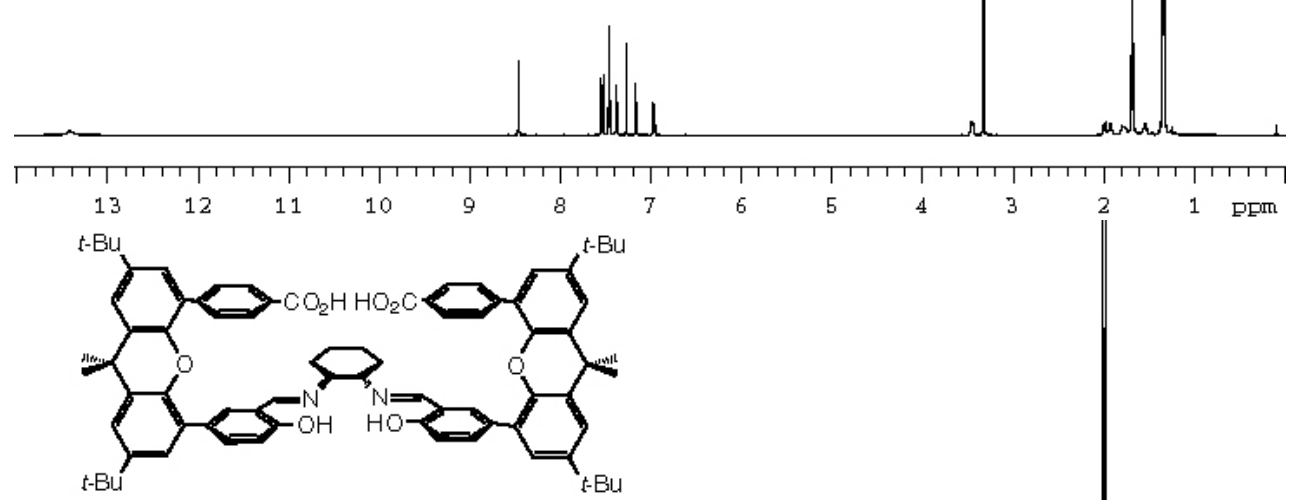

15

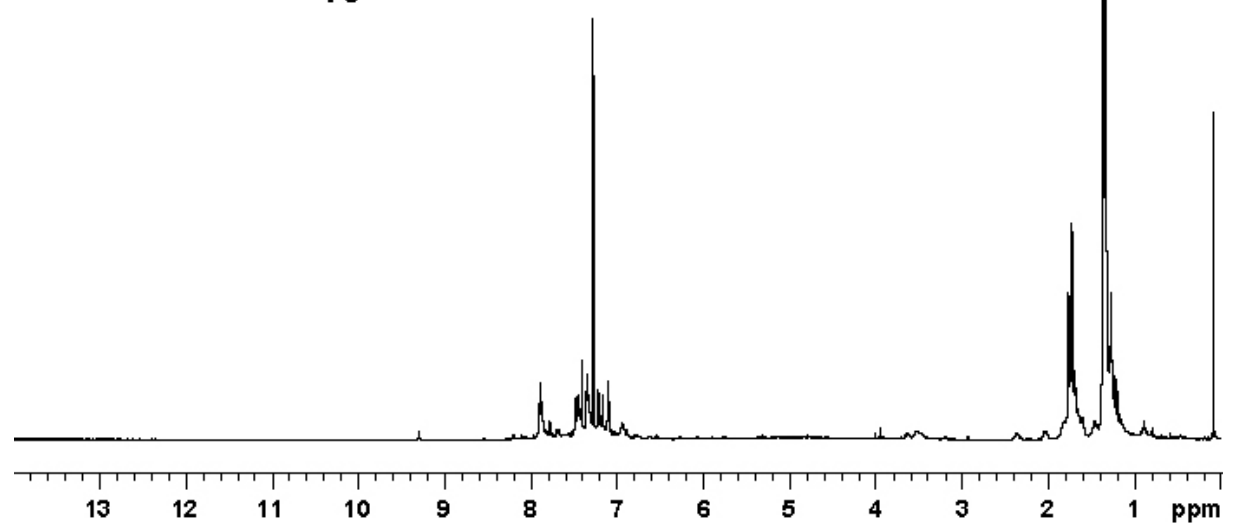




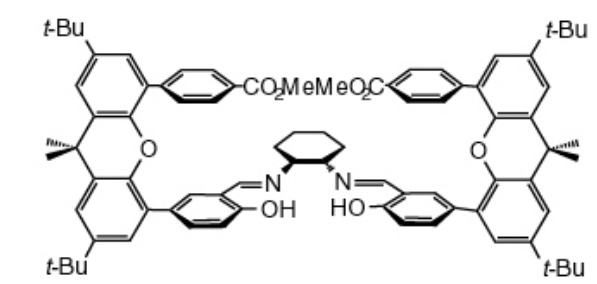

16

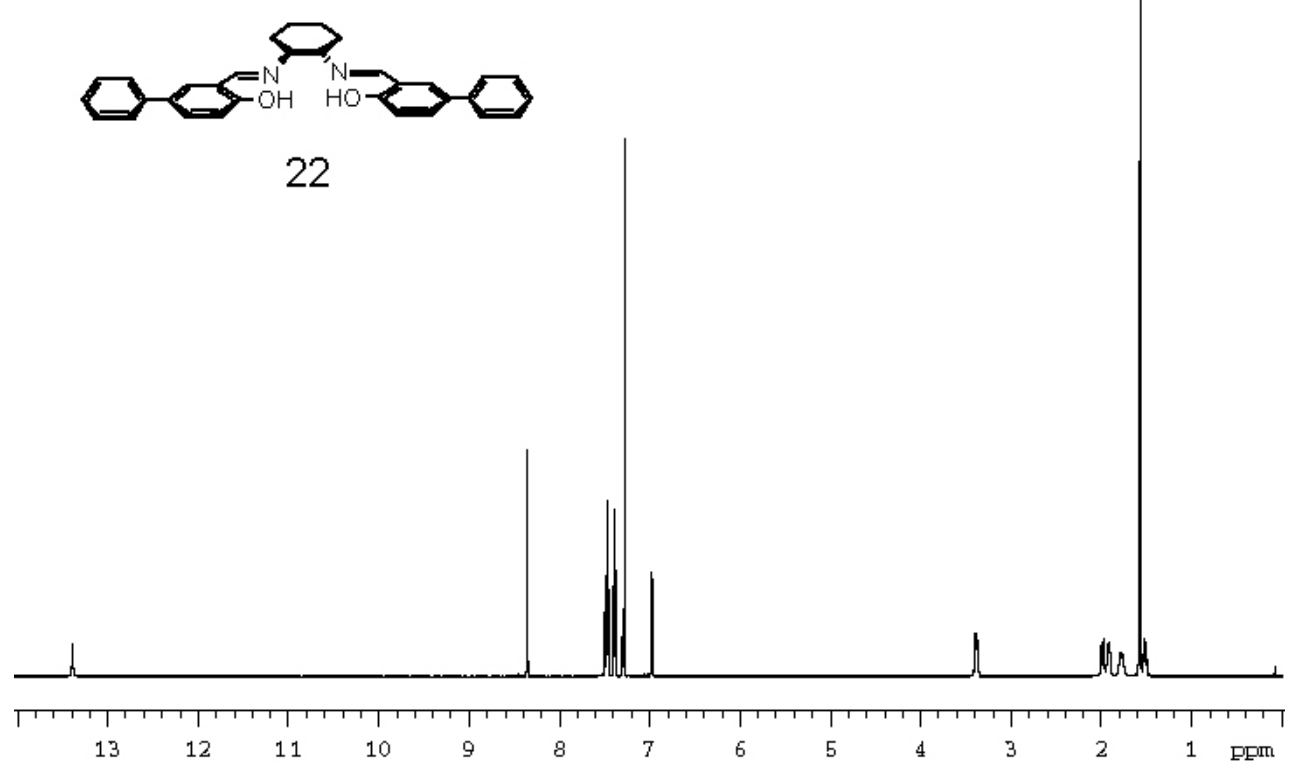




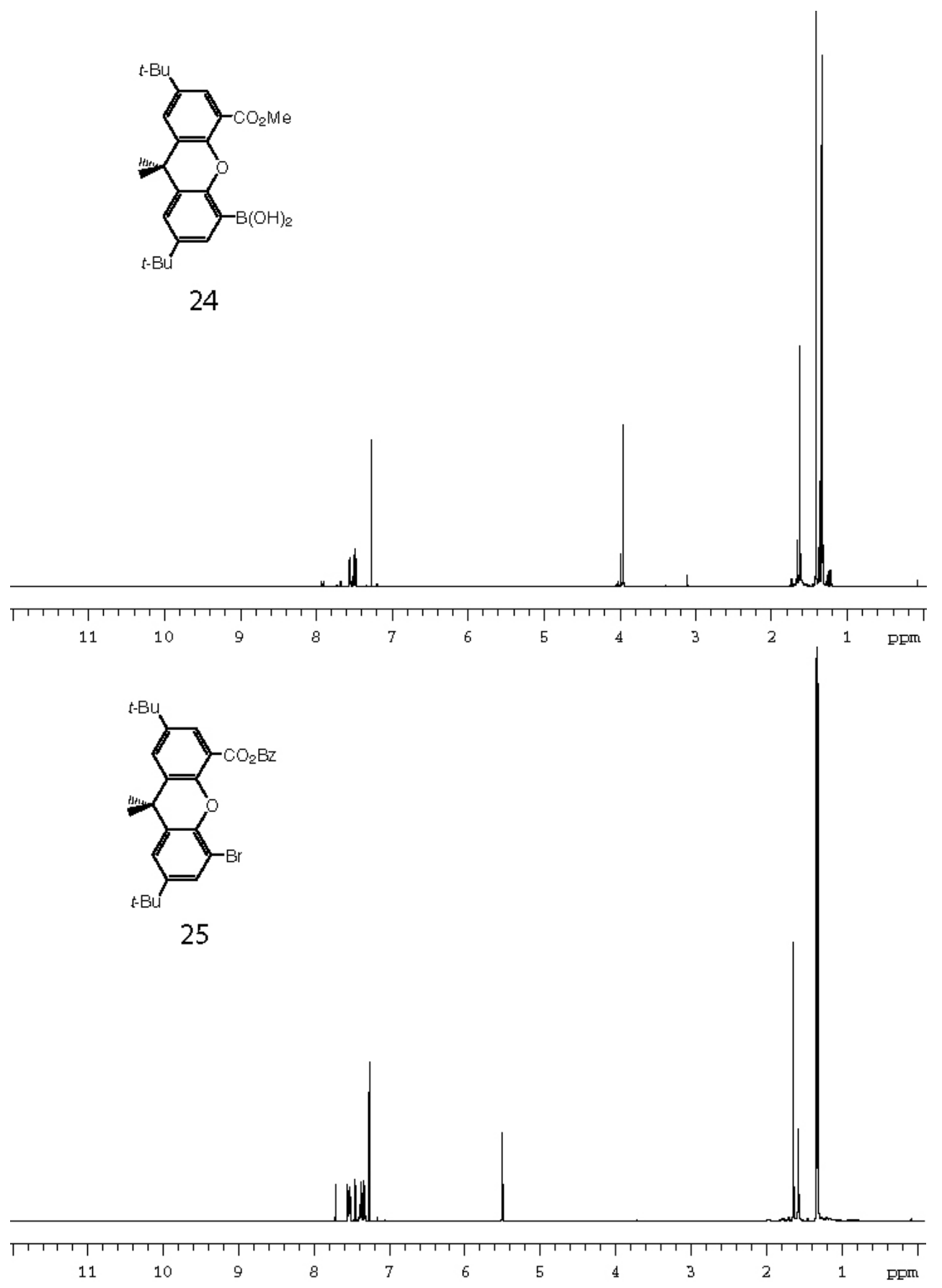




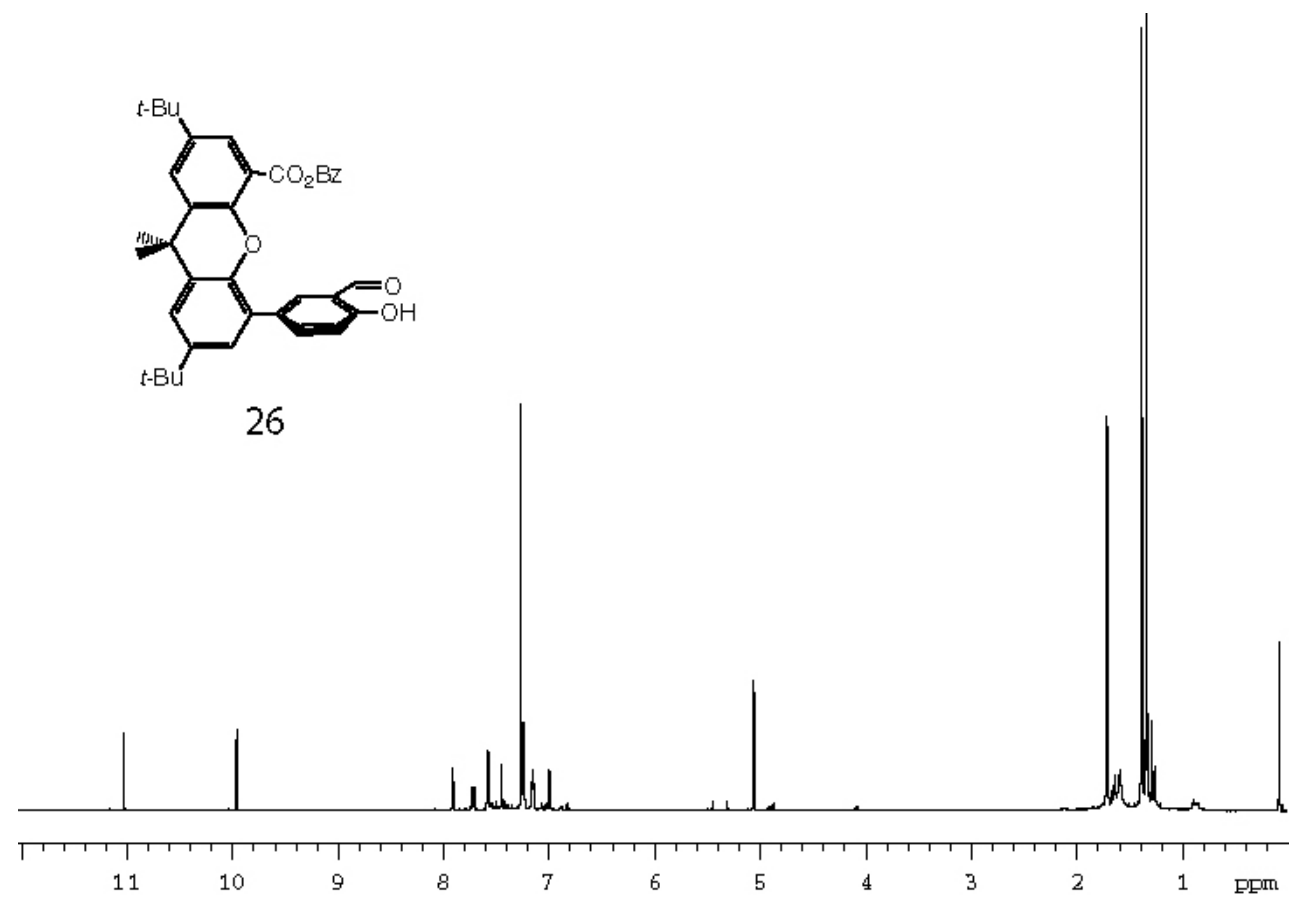

